\title{
Review of Classical Analytical Results for the Motion of a Rydberg Electron around a Polar Molecule under Magnetic or Electric Fields of Arbitrary Strengths in Axially Symmetric Configurations
}

Eugene Oks

check for updates

Citation: Oks, E. Review of Classical Analytical Results for the Motion of a Rydberg Electron around a Polar Molecule under Magnetic or Electric Fields of Arbitrary Strengths in Axially Symmetric Configurations. Symmetry 2021, 13, 2171. https:// doi.org/10.3390/sym13112171

Academic Editor: Ulrich

D. Jentschura

Received: 8 October 2021

Accepted: 9 November 2021

Published: 12 November 2021

Publisher's Note: MDPI stays neutral with regard to jurisdictional claims in published maps and institutional affiliations.

Copyright: (C) 2021 by the author Licensee MDPI, Basel, Switzerland. This article is an open access article distributed under the terms and conditions of the Creative Commons Attribution (CC BY) license (https:// creativecommons.org/licenses/by/ $4.0 /)$.
Physics Department, 380 Duncan Drive, Auburn University, Auburn, AL 36849, USA; goks@physics.auburn.edu

\begin{abstract}
We review classical studies of the oscillatory-precessional motion of an electron in the field of an electric dipole (the latter representing the polar molecule) with or without external magnetic or electric fields. The focus is on the most recent studies. In one study (at zero external field), it was shown that, generally, the oscillations being in the meridional direction and the precession being along parallels of latitude can take place on the same time scale-contrary to the statement from the previous literature. In another study, it was shown that a magnetic field enables new ranges of the bound oscillatory-precessional motion of the Rydberg electron and that in one of the new ranges, the period of the $\theta$-oscillations has the non-monotonic dependence on primary parameter of the system. This is a counterintuitive result. In yet another study, it was shown that under the electric field there are two equilibrium circular states of a positive energy and one equilibrium state of a negative energy. The existence of the equilibrium state of the negative energy is a counterintuitive result since at the absence of the field, the bound state was possible only for the zero energy. Thus, it is a counterintuitive result that in this case the electric field can play the role of a stabilizing factor.
\end{abstract}

Keywords: polar molecule; Rydberg electron; axial symmetry; magnetic field; stabilization by electric field; classical motion

\section{Introduction}

The motion of an electron in the field of a polar molecule is one of the most fundamental problems in atomic and molecular physics - on par with the problem of a hydrogen atom. If the dimension of the molecule is much smaller than its separation from the electron, the molecule can be considered as the point-like dipole. Obviously, such system possesses the axial symmetry - just from the geometrical considerations. Fermi and Teller [1] were the first to study a mathematically an equivalent problem in 1947: the motion of a low-energy muon in the vicinity of a hydrogen atom. In the subsequent years, there were lots of other relevant studies-see, e.g., paper [2] and references therein.

When the electron is only weakly bound to the dipole, i.e., the electron is in the Rydberg state, the classical treatment is appropriate. For the case of the finite electric dipole, the following two works have been published. In paper [3], the authors provided an analytical treatment of this problem by using the elliptical coordinates $(\xi, \eta, \phi)$. They demonstrated that the bound motion is possible in some finite region in terms of the coordinates $\xi$ and $\eta$ for any finite dipole. The region has the shape of a torus of the revolution with respect to the axis of the dipole.

In paper [4], the authors analyzed the motion of a negative charge around a finite dipole by using the cylindrical coordinates. First, they investigated circular orbits where the negative charge revolves in a plane orthogonal to the dipole. (A particular application described in detail in paper [4] was for the motion of a muon in the field of a finite dipole consisting of a stationary proton and electron, but their mathematical results are also valid for the motion of an electron around any finite dipole.) The authors of paper [4] demonstrated the existence of stable circular orbits under the condition that the dipole size 
exceeds some threshold value, which coincided with the corresponding threshold value obtained in quantum-mechanics-based investigations of this system-see, e.g., paper [2] and references therein. Then, they removed the assumption of circular orbits and showed the existence of stable conic-helical orbits of the negative charge in such systems. The orbit is located on the surface of a cone (specifically, on a right frustum of the cone), the cone axis coinciding with the dipole axis.

It is important to emphasize the following. An electron around a finite dipole is a particular case of the general problem: a charge moving around two stationary Coulomb centers. This general problem possesses a higher than geometrical symmetry-the algebraic symmetry. The manifestation of this algebraic symmetry is the existence of an additional integral of the motion: the projection of the super-generalized Runge-Lenz vector on the geometrical axis of symmetry, the super-generalized Runge-Lenz vector being derived in paper [5].

For the situation where the dipole can be considered point-like (i.e., where the separation of the electron from the dipole is much greater than the dipole size, the corresponding classical motion was first studied in paper [6] in 1968. The author of paper [6] demonstrated that only for the zero total energy of the electron the bound motion can happen, and it occurs on the surface of a sphere. Specific analytical results were developed in paper [6] to the form of integrals, but no integration has been performed and no qualitative description of the classical motion was given (see some details below).

Later, in paper [7], the author considered on semicircular meridional orbits on a sphere. He assumed the light particle to be charged positively and noted that in this limiting case, the situation is mathematically identical to a pendulum.

Then, in paper [8], the author concentrated on the following two types of orbits. One of them was the same as considered in paper [7]. Another one was a circular orbit coinciding with some specific parallel of latitude. The author of paper [8] noted that in the general case, the motion is a combination of relatively large oscillations in terms of the polar angle $\theta$ and a relatively slow precession along the azimuthal angle $\varphi$.

The most detailed classical analytical solution for the motion of an electron in the field of a point-like dipole was presented in paper [9]. The author of paper [9] showed that, in the general case, the oscillations in the $\theta$-direction and the precession in the $\varphi$-precessions can actually happen on the same time scale-consequently, the opposite statement from paper [8] was erroneous.

The author of paper [9] obtained numerous specific analytical results for a variety of particular cases. He also deduced a condition, under which the electron motion (being in general conditionally periodic) can turn out to be periodic, manifested by a closed trajectory of the electron. Details are presented in Section 2.

In Section 3, we present a more complicated problem where the system is subjected to a magnetic field $\mathbf{B}$ parallel or antiparallel to the dipole. This configuration retains the axial symmetry. Analytical results are obtained for an arbitrary strength of the magnetic field. The magnetic field enables additional ranges of the bound motion of the electron. In one of the additional ranges of the oscillatory-precessional motion, it turns out that the dependence of the period of the meridional oscillations on the primary parameter of the system is non-monotonic, which is a counterintuitive result.

In Section 4, we present the study of the situation where instead of the magnetic field, there is an electric field $\mathbf{F}$ parallel or antiparallel to the electric dipole. This configuration also retains the axial symmetry. Again, analytical results are obtained for an arbitrary strength of the electric field. The electric field makes possible one equilibrium state of a negative energy and two equilibrium states of a positive energy. While at the absence of the electric field the bound state existed only for the zero energy, the possibility of the equilibrium state of the negative energy constitutes a counterintuitive result. Indeed, in this case, the electric field is a stabilizing factor, while, usually, the electric field promotes the ionization of atomic systems. We provide analytical expressions for the equilibrium radius of the electron orbit, the polar angle of the orbit, and the electron revolution frequency. 
Since the frequency of spectral lines emitted by the Rydberg electron coincides with the revolution frequency, then these analytical results make the experimental determination of the energy of the equilibrium states possible.

\section{Classical Non-Circular Orbits at the Absence of External Fields}

Following paper [9], the energy of the electron in the field of the point-like electric dipole (of the dipole moment $\mathrm{D}$ ) located at the origin of the spherical polar coordinate reference frame (the z-axis being along the dipole) can be expressed as:

$$
\mathrm{E}=\mathrm{m}\left[(\mathrm{dr} / \mathrm{dt})^{2}+\mathrm{r}^{2}(\mathrm{~d} \theta / \mathrm{dt})^{2}+\mathrm{r}^{2} \sin ^{2} \theta(\mathrm{d} \phi / \mathrm{dt})^{2}\right] / 2-\mathrm{eD} \cos \theta / \mathrm{r}^{2},
$$

where e and $\mathrm{m}$ are the absolute value of the electron charge and the electron mass, respectively. The energy does not depend on $\varphi$, so that the corresponding generalized momentum $\mathrm{p}_{\varphi}$ (which is the z-projection of the angular momentum $\mathrm{M}_{\mathrm{z}}$ of the electron) is conserved:

$$
\mathrm{p}_{\phi}=\mathrm{mr}^{2} \sin ^{2} \theta(\mathrm{d} \phi / \mathrm{dt})=\text { const. }
$$

Of course, this is the consequence of the axial symmetry of the configuration under the consideration, and it enables the separation of the $\theta$ - and $\phi$-motions.

In paper [6], it was demonstrated that the bound motion can occur only if $E=0$ and $r=$ const. In other words, the electron is confined to a sphere: only $\theta$ and $\phi$ remain the dynamical variables.

The differential equation for the $\theta$-motion derived in paper [6] is as follows:

$$
(d x / d t)^{2}=\left[2 e D /\left(m r^{4}\right)\right]\left(-x^{3}+x-K\right) .
$$

Here:

$$
\mathrm{x}=\cos \theta, \quad \mathrm{K}=\mathrm{p}_{\varphi}{ }^{2} /(2 \mathrm{meD}) .
$$

From Equation (2) follows the differential equation for the $\phi$-motion:

$$
\mathrm{d} \phi / \mathrm{dt}=\mathrm{p}_{\phi} /\left(\mathrm{mr}^{2} \sin ^{2} \theta\right)=\mathrm{p}_{\phi} /\left[\mathrm{mr}^{2}\left(1-\mathrm{x}^{2}\right)\right] .
$$

So, the sequence of tasks can be to find $x(t)$ from Equation (3) and then obtain $\phi(t)$ from Equation (5).

For the bound motion to be possible, the following polynomial

$$
y(x)=-x^{3}+x-K
$$

in Equation (3) should be positive in some segment of the variable $\mathrm{x}$ within the interval from -1 to 1 , as noted in paper [6]. The polynomial has a maximum equal to $-K+2 / 3^{3 / 2}$ at $x=1 / 3^{1 / 2}$ and a negative minimum equal to $-K-2 / 3^{3 / 2}$ at $x=-1 / 3^{1 / 2}$. The necessary condition for the existence of the bound motion is for the maximum to be positive. This condition translates into the following requirement [6]:

$$
\mathrm{K}<2 / 3^{3 / 2}=\mathrm{K}_{\max }
$$

or equivalently (according to Equation (4)):

$$
\mathrm{D}>3^{3 / 2} \mathrm{p}_{\phi}^{2} /(4 \mathrm{me}) .
$$

Under condition (7), the x-motion (i.e., the $\theta$-motion) could be confined between two positive turning points. In other words, the bound motion can happen only in the upper hemisphere [6]. 
The results below have been obtained in paper [9]. The positive turning points $x_{2}$ and $x_{3}\left(x_{3}>x_{2}\right)$ of the motion in the $x$-coordinate are the real roots of the cubic equation:

$$
x^{3}-x+K=0 .
$$

The explicit expressions for these roots are the following:

$$
\begin{array}{r}
\mathrm{x}_{2}(\mathrm{~K})=(-1)^{2 / 3} 2^{1 / 3} /\left[\left(729 \mathrm{~K}^{2}-108\right)^{1 / 2}-27 \mathrm{~K}\right]^{1 / 3}-(-1)^{1 / 3}\left[\left(729 \mathrm{~K}^{2}-108\right)^{1 / 2}-27 \mathrm{~K}\right]^{1 / 3} /\left(2^{1 / 3} 3\right) . \\
\mathrm{x}_{3}(\mathrm{~K})=2^{1 / 3} /\left[\left(729 \mathrm{~K}^{2}-108\right)^{1 / 2}-27 \mathrm{~K}\right]^{1 / 3}-\left[\left(729 \mathrm{~K}^{2}-108\right)^{1 / 2}-27 \mathrm{~K}\right]^{1 / 3} /\left(2^{1 / 3} 3\right) .
\end{array}
$$

In Figure 1, there are plots of both $\mathrm{x}_{2}(\mathrm{~K})$ and $\mathrm{x}_{3}(\mathrm{~K})$. Specifically, $\mathrm{x}_{2}(\mathrm{~K})$ is the lower part of the double-valued curve, and $x_{3}(\mathrm{~K})$ is the upper part of the double-valued curve. These two parts intersect at $K=K_{\max }=2 / 3^{3 / 2}$, where $x_{2}\left(2 / 3^{3 / 2}\right)=x_{3}\left(2 / 3^{3 / 2}\right)=1 / 3^{1 / 2}$.

\section{Roots}

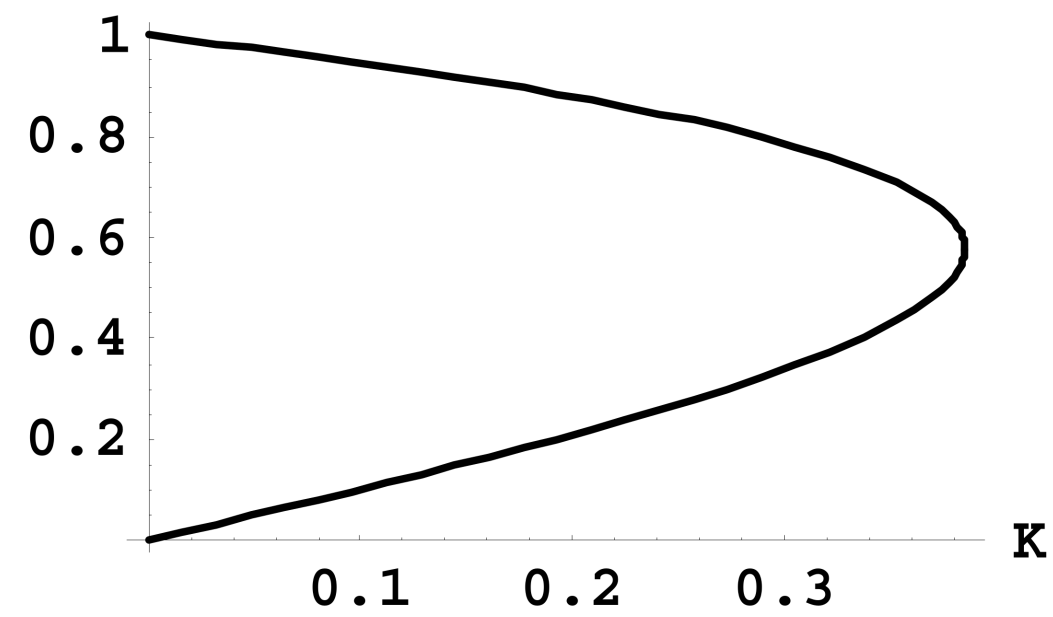

Figure 1. Positive roots $x_{2}$ and $x_{3}$ of the cubic Equation (9) versus $K=p_{\phi}{ }^{2} /(2 m e D)$ : $(K)$ and $x_{3}(K)$ are the lower part and the upper part of the double-valued curve, respectively [9].

After introducing a scaled dimensionless time $\tau$ as follows:

$$
\tau=\mathrm{t}\left[2 \mathrm{eD} /\left(\mathrm{mr}^{4}\right)\right]^{1 / 2},
$$

Equation (3) can be rewritten as:

$$
\mathrm{d} \tau= \pm \mathrm{dx} /\left(-\mathrm{x}^{3}+\mathrm{x}-\mathrm{K}\right)^{1 / 2} .
$$

Both the plus and minus signs should be considered in Equation (13)—contrary to the choice of only the minus sign in Equation (12) of paper [6].

Now we present important particular cases. For $K=K_{\max }=2 / 3^{3 / 2}$, the electron executes a circular orbit representing the parallel of latitude that corresponds to $\cos \theta=1 / 3^{1 / 2}$, so that $\theta=0.9553$ and $\mathrm{rad}=54.74$ degrees. The angular velocity of the electron is constant (see Equation (5)):

$$
\mathrm{d} \phi / \mathrm{dt}=\mathrm{p}_{\phi} /\left(2 \mathrm{mr}^{2}\right),
$$

so that the period is:

$$
\mathrm{T}=4 \pi m r^{2} /\left(3 \mathrm{p}_{\phi}\right) .
$$


According to Equation (4), $\mathrm{p}_{\phi}=(2 \mathrm{KmeD})^{1 / 2}$, meaning that for $\mathrm{K}=\mathrm{K}_{\max }=2 / 3^{3 / 2}$, one has $\mathrm{p}_{\phi}=2(\mathrm{meD})^{1 / 2} / 3^{3 / 4}$. Then, Equations (13) and (14) can be represented in the following forms:

$$
\mathrm{d} \phi / \mathrm{dt}=3^{1 / 4}\left[\mathrm{eD} /\left(\mathrm{mr}^{4}\right]^{1 / 2}, \quad \mathrm{~T}=\left(2 \pi / 3^{1 / 4}\right)\left[\mathrm{mr}^{4} /(\mathrm{eD})\right]^{1 / 2} .\right.
$$

Next, in the case of $K<<1$, Equations (9) and (10) can be rewritten as follows:

$$
\mathrm{x}_{2}(\mathrm{~K}) \approx \mathrm{K}, \quad \mathrm{x}_{3}(\mathrm{~K}) \approx 1-\mathrm{K} / 2 .
$$

In the particular case of $K=0$ (that is, $p_{\phi}=0$ ), the electron oscillates along a semicircle. The semicircle is positioned in a meridional plane in the upper hemisphere. In this particular case, Equation (13) can be integrated analytically. The result is the explicit dependence of the scaled time $\tau$ on $x$ (i.e., on $\cos \theta)$ :

$$
\tau=-\left\{ \pm 2 \mathrm{i} F\left[\arcsin (-\mathrm{x})^{1 / 2}, \quad-1\right]\right\},
$$

$\mathbf{F}(\alpha, q)$ being the incomplete elliptic integral of the first kind. The motion occurs in the range of $x$ from 0 to 1 . In this range, the right side of Equation (18) is real-despite the presence of the imaginary unit $\mathrm{i}$ in Equation (18). For $\mathrm{x}<<1$, Equation (18) yields:

$$
\tau \approx \pm 2 x^{1 / 2}
$$

(We note that there was a misprint in the corresponding Equation (19) from paper [9].)

Figure 2 shows the time evolution of the electron as it moves from a point on the equator $(x=0$ at $\tau=0)$ to the north pole $(x=1$ at $\tau=2.622)$, then changes its geographical longitude of in the upper hemisphere by 180 degrees and moves to the equator $(x=0$ at $\tau=5.244)$, then back to the north pole $(x=1$ at $\tau=7.866)$, then changes again its geographical longitude of in the upper hemisphere by 180 degrees and moves to the equator ( $\mathrm{x}=0$ at $\tau=10.488$ ). (We note that in paper [9], the corresponding time evolution was not described accurately.)

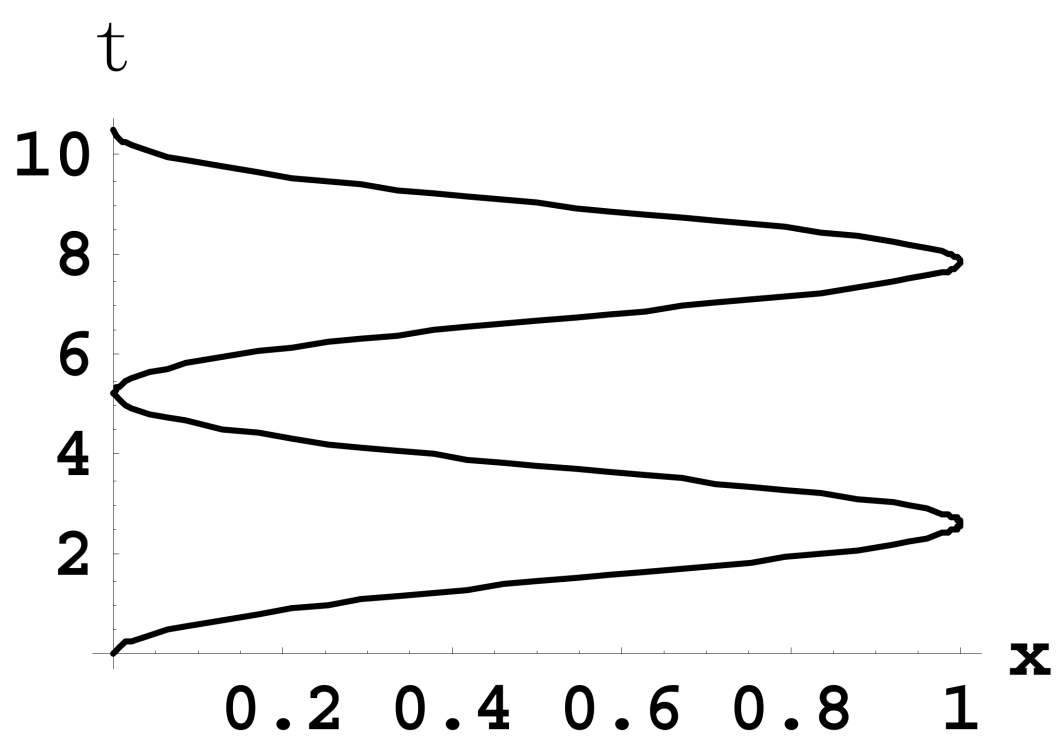

Figure 2. The time evolution of the electron as it oscillates along a semicircle through the north pole of the upper hemisphere $(\mathrm{K}=0)$.

The full period of oscillations is equal to:

$$
\tau_{0}=4 \tau(1)=10.488
$$


or:

$$
\mathrm{T}=10.488\left[\mathrm{mr}^{4} /(2 \mathrm{eD})\right]^{1 / 2}
$$

in the usual units.

For the general case of an arbitrary $K$ from the segment $\left[0, K_{\max }=2 / 3^{3 / 2}\right]$, the scaled time depends on $\mathrm{x}$ as follows:

$$
\tau(\mathrm{x}, \mathrm{K})= \pm \int_{\mathrm{x} 2(\mathrm{~K})}^{\mathrm{x}} \quad \mathrm{dz} /\left(-\mathrm{z}^{3}+\mathrm{z}-\mathrm{K}\right)^{1 / 2}
$$

the lower limit of the integration being the smaller of the two turning points. In Figure 3, the time evolution versus $x$ during one period of the $\theta$-motion, as $x$ changes from $x_{2}(K)$ to $\mathrm{x}_{3}(\mathrm{~K})$ and back to $\mathrm{x}_{2}(\mathrm{~K})$ is presented for $\mathrm{K}=0.3$ (dotted line), $\mathrm{K}=0.2$ (dashed line), and $\mathrm{K}=0.1$ (solid line).

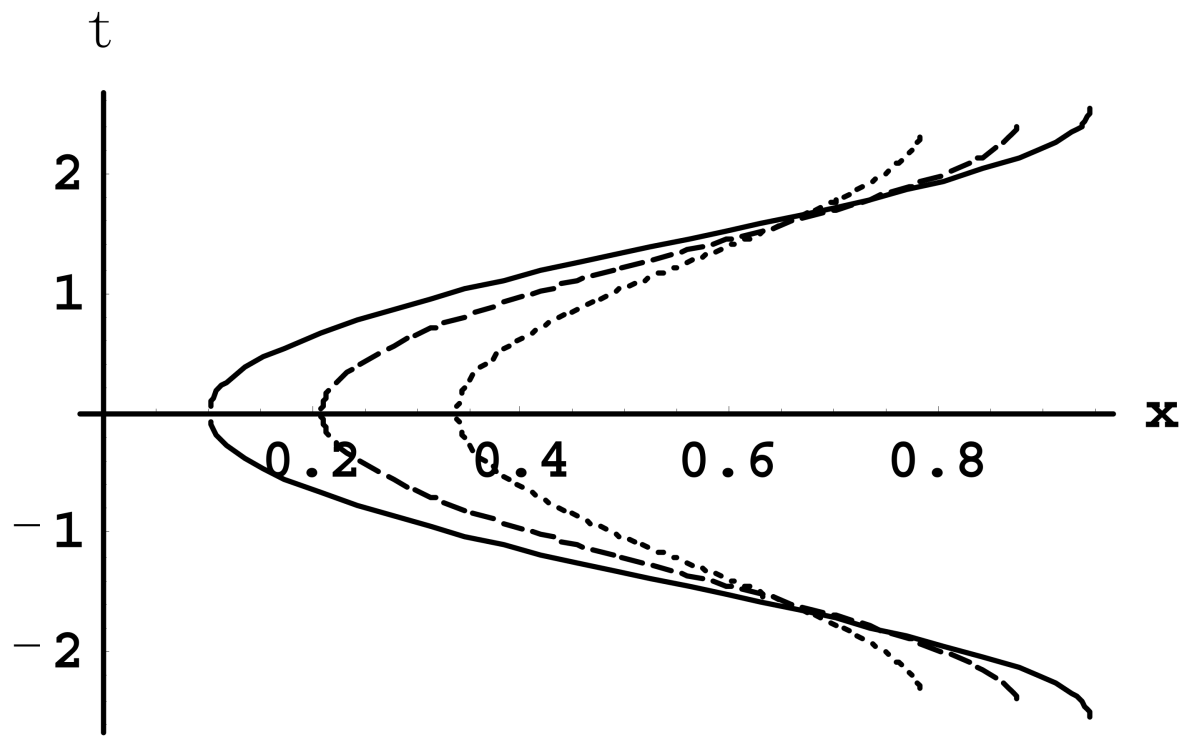

Figure 3. The scaled time $\tau$ versus $x=\cos \theta$ during one period of the $\theta$-motion for $K=0.3$ (dotted line), $\mathrm{K}=0.2$ (dashed line), and $\mathrm{K}=0.1$ (solid line) [9].

The integral in Equation (22) can be calculated analytically for $\mathrm{K}<<1$ by expanding it in Taylor series up to the terms $\sim \mathrm{K}$ :

$$
\tau(\mathrm{x}, \mathrm{K}) \approx \pm\left\{\mathrm{f}(\mathrm{x})-\mathrm{f}\left[\mathrm{x}_{2}(\mathrm{~K})\right]+\mathrm{Kg}(\mathrm{x})-\mathrm{Kg}\left[\mathrm{x}_{2}(\mathrm{~K})\right]\right\},
$$

where:

$$
\begin{aligned}
& f(x)=-2 i F\left[\arcsin (-x)^{1 / 2}, \quad-1\right], \\
& g(x)=\left[3 x^{2}-2-x^{2}\left(1-x^{2}\right)^{1 / 2}{ }_{2} F_{1}\left(3 / 4,1 / 2,7 / 4, x^{2}\right)\right] /\left[2\left(x-x^{3}\right)^{1 / 2}\right] .
\end{aligned}
$$

In Equation (25), ${ }_{2} \mathrm{~F}_{1}(\mathrm{a}, \mathrm{b}, \mathrm{c}, \mathrm{z})$ is the hypergeometric function. The accuracy of this approximate analytical result for $\tau(x, K)$ is demonstrated in Figure 4 for $K=0.01$ (solid line), where it is compared to the corresponding exact result obtained by the numerical integration in Equation (22) (dashed line). The accuracy of the analytical result is very good, as shown in Figure 4. 


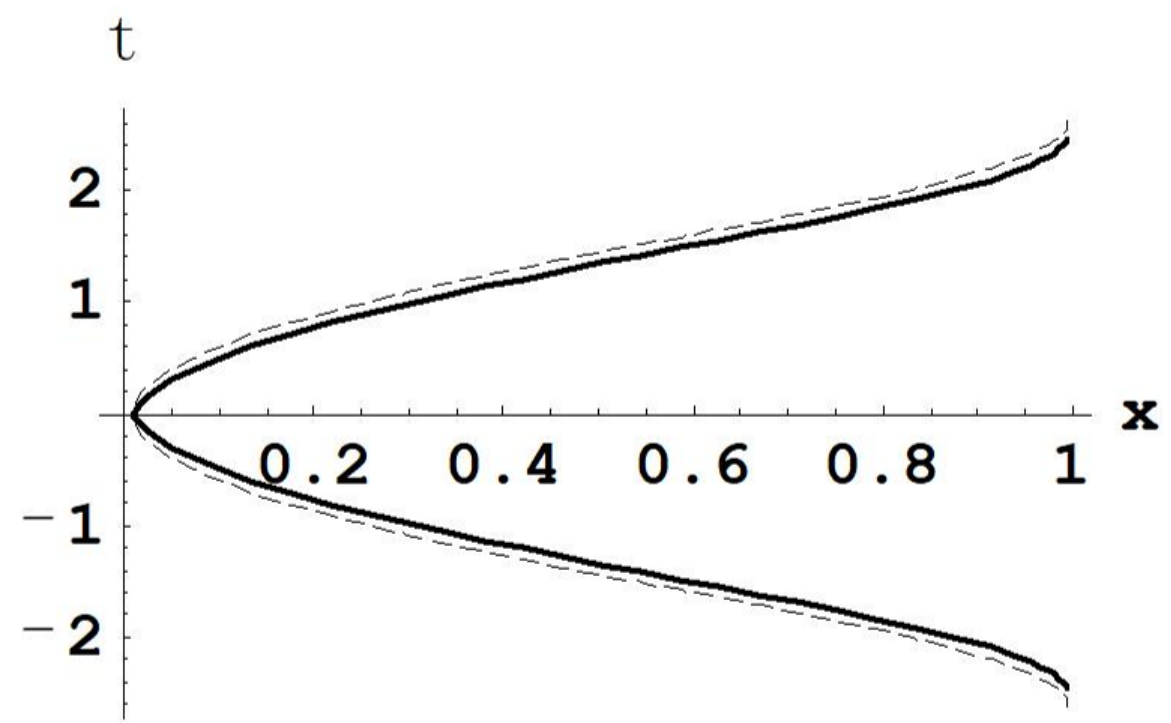

Figure 4. Comparison of the exact result for $\tau(x, K)$ obtained by the numerical integration in Equation (22) (dashed line) with the corresponding approximate analytical result from Equation (23) for $\mathrm{K}=0.01$ (solid line) [9].

The scaled period $\mathrm{T}_{\theta}$ of the $\theta$-motion can be represented as follows (in units of $\left.\mathrm{mr}^{4} /(2 \mathrm{eD})\right)$ :

$$
\mathrm{T}_{\theta}(\mathrm{K})=2 \quad \int_{\mathrm{x} 2(\mathrm{~K})}^{\mathrm{x} 3(\mathrm{~K})} \mathrm{dz} /\left(-\mathrm{z}^{3}+\mathrm{z}-\mathrm{K}\right)^{1 / 2} .
$$

The scaled period $\mathrm{T}_{\theta}$ versus $\mathrm{K}$ is displayed in Figure 5.

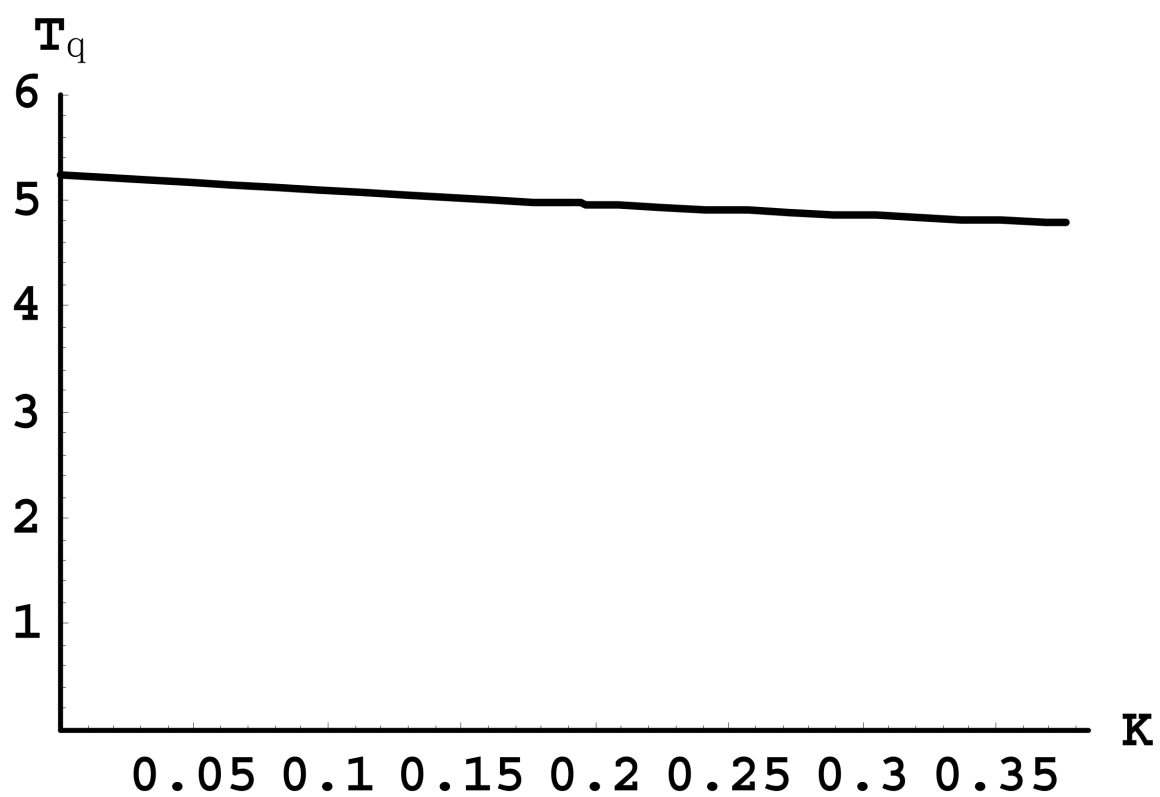

Figure 5. The scaled period $T_{\theta}$ of the $\theta$-motion versus the parameter $K=p_{\varphi}{ }^{2} /(2 m e D)$ : The period $\mathrm{T}_{\theta}$ is in units of $\mathrm{mr}^{4} /(2 \mathrm{eD})[9]$.

An explicit analytical result for the scaled period of the $\theta$-motion can be obtained for $\mathrm{K}<<1$, as follows:

$$
\mathrm{T}_{\theta}(\mathrm{K}) \approx 2\{\mathrm{f}(1-\mathrm{K} / 2)-\mathrm{f}(\mathrm{K})+\mathrm{Kg}(1-\mathrm{K} / 2)-\mathrm{Kg}(\mathrm{K})\},
$$

where functions $\mathrm{f}$ and $\mathrm{g}$ are defined by Equations (24) and (25), respectively. 
Next is the analysis of the $\phi$-motion. Equation (5) can be represented as follows:

$$
\mathrm{d} \phi=\mathrm{p}_{\phi} \mathrm{dt} /\left[\mathrm{mr}^{2}\left(1-\mathrm{x}^{2}\right)\right] .
$$

Then, the dependence of the angular variable $\varphi$ on the angular variable $x=\cos \theta \operatorname{can}$ be derived by using the relation between $\mathrm{dt}$ and $\mathrm{dx}$ from Equation (3):

$$
\phi(\mathrm{K}, \mathrm{x})=\mathrm{K}^{1 / 2} \quad \int_{\mathrm{x} 2(\mathrm{~K})}^{\mathrm{x}} \quad \mathrm{dz} /\left[\left(1-\mathrm{z}^{2}\right)\left[\left(-\mathrm{z}^{3}+\mathrm{z}-\mathrm{K}\right)^{1 / 2}\right] .\right.
$$

Figure 6 shows $\phi$ versus $x$ during one half-period of the $\theta$-motion for $\mathrm{K}=0.1$ (solid line), $\mathrm{K}=0.2$ (dashed line), and $\mathrm{K}=0.3$ (dotted line). One can see that the curve $\phi(\mathrm{x})$ becomes steeper and the change of $\phi$ over one half-period of the $\theta$-motion slightly increases with the growth of the parameter $\mathrm{K}$.

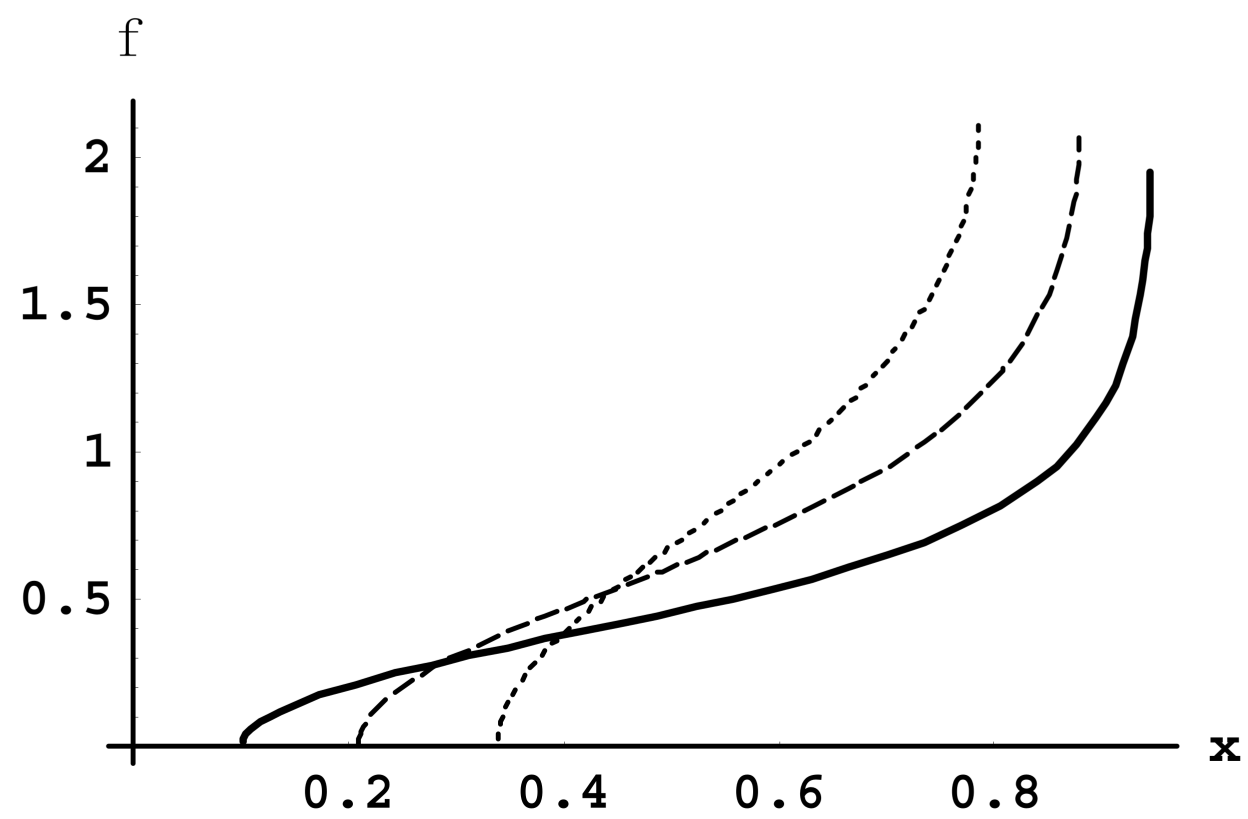

Figure 6. The angular variable $\phi$ versus $x=\cos \theta$ during one half-period of the $\theta$-motion for $K=0.1$ (solid line), $\mathrm{K}=0.2$ (dashed line), and $\mathrm{K}=0.3$ (dotted line).

The following explicit analytical result for $\phi(K, x)$ can be derived for $K<<1$ :

$$
\phi(\mathrm{K}, \mathrm{x}) \approx \mathrm{K}^{1 / 2}[\mathrm{j}(\mathrm{x})-\mathrm{j}(\mathrm{K})],
$$

where:

$$
j(x)=\left[x /\left(1-x^{2}\right)\right]^{1 / 2}\left\{1-(x-1 / x)^{1 / 2} \mathbf{F}\left[\operatorname{arccsc}\left(x^{1 / 2}\right),-1\right]\right\} .
$$

In Equation (31), $\mathbf{F}(\alpha, \mathrm{q})$ is the incomplete elliptic integral of the first kind. In Equation (30), the fact that $\mathrm{x}_{2}(\mathrm{~K}) \approx \mathrm{K}$ for $\mathrm{K}<<1$ has been used.

The accuracy of the approximate analytical result for $\phi(K, x)$ from Equation (30) is demonstrated in Figure 7 for $K=0.02$ (solid line), where it is compared to the corresponding exact result from Equation (29) (dashed line). The accuracy of the analytical result is very good, as shown. 


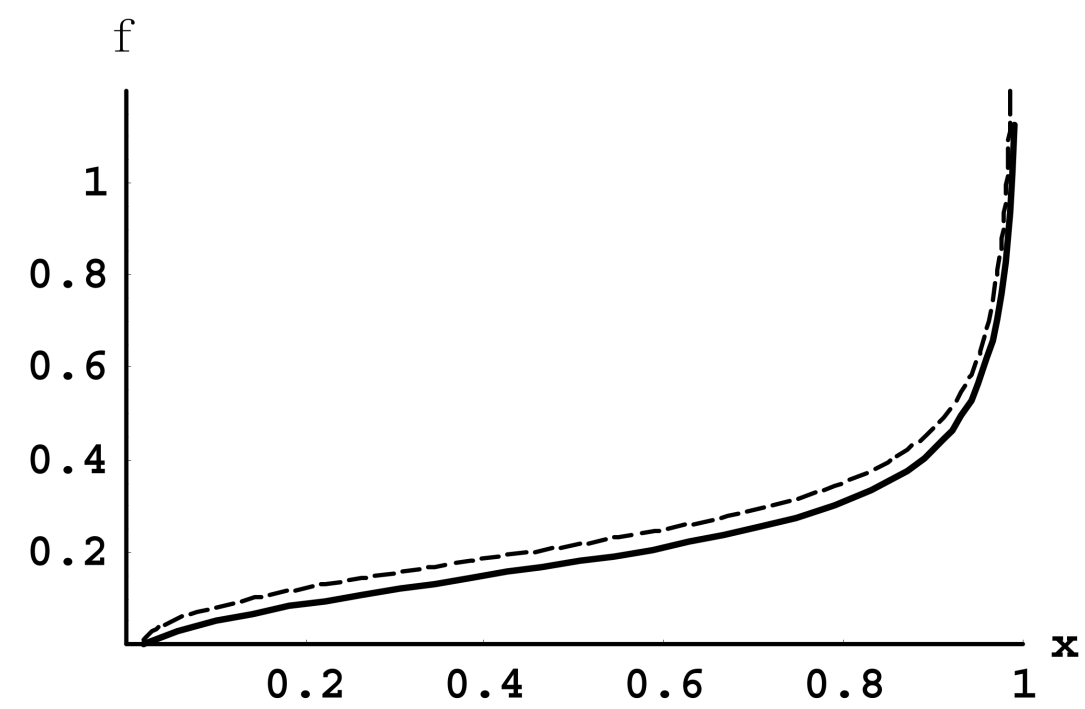

Figure 7. Comparison of the exact result for $\phi(K, x)$ from Equation (29), shown by the dashed line, with the corresponding approximate analytical result from Equation (30), shown by the solid line, for $\mathrm{K}=0.02$ [9].

During one half-period of the $\theta$-motion, the angular variable $\phi$ changes by the following amount:

$$
\Delta \phi(\mathrm{K})=\mathrm{K}^{1 / 2} \int_{\mathrm{x} 2(\mathrm{~K})}^{\mathrm{x} 3(\mathrm{~K})} \mathrm{dz} /\left[\left(1-\mathrm{z}^{2}\right)\left[\left(-\mathrm{z}^{3}+\mathrm{z}-\mathrm{K}\right)^{1 / 2}\right] .\right.
$$

In the case where $\mathrm{K}$ is close to zero, one has $\Delta \phi \approx \pi / 2$, while for the case where $\mathrm{K}$ is close to $\mathrm{K}_{\max }=2 / 3^{3 / 2}$, one has $\Delta \varphi \approx \pi / 2^{1 / 2}$, so that:

$$
1 / 2<\Delta \phi / \pi<1 / 2^{1 / 2}
$$

The quantity $\Delta \phi$ monotonically increases between these two limits as K increases.

In the general case, the precession of the electron along parallels of latitude is combined with the oscillations in the upper hemisphere in the meridional direction. In general, the amount $\Delta \varphi(\mathrm{K})$, by which the angle $\phi$ advances during one period of the $\theta$-oscillation, is not equal to $n \pi / m$, where $n$ and $m$ are relatively small integers. Therefore, in the general case, the electron trajectory is not a closed curve: The motion is conditionally periodic. However, in some special cases, where

$$
\Delta \phi(\mathrm{K})=\mathrm{n} \pi / \mathrm{m},
$$

the motion becomes periodic. Here are examples corresponding to the three lowest pairs of integers $n$ and $m$ in Equation (34): $(n=2, m=3),(n=3, m=5)$, and $(n=4, m=7)$. Figure 8 shows the intersections of the curve $\Delta \phi(\mathrm{K}) / \pi$ with the horizontal lines, corresponding to $\mathrm{n} / \mathrm{m}$, for the above three pairs.

Below are the details for all three cases. For $n=2, m=3$, the angle $\phi$ completes 2 full circles during 3 periods of the $\theta$-oscillations. This occurs for $\mathrm{K}=0.2103$. The corresponding frequency of the $\theta$-oscillations is 1.2703 in units of $2 \mathrm{eD} /\left(\mathrm{mr}^{4}\right)$. In this case, the intensity of the spectral line emitted at the frequency of 2.5406 in units of $2 \mathrm{eD} /\left(\mathrm{mr}^{4}\right)$, which is the double-frequency of the $\theta$-oscillations, would be enhanced (compared to the intensity of the spectral line at the primary frequency of the $\theta$-oscillations) due to the resonance with the triple-frequency of the $\varphi$-precession. 


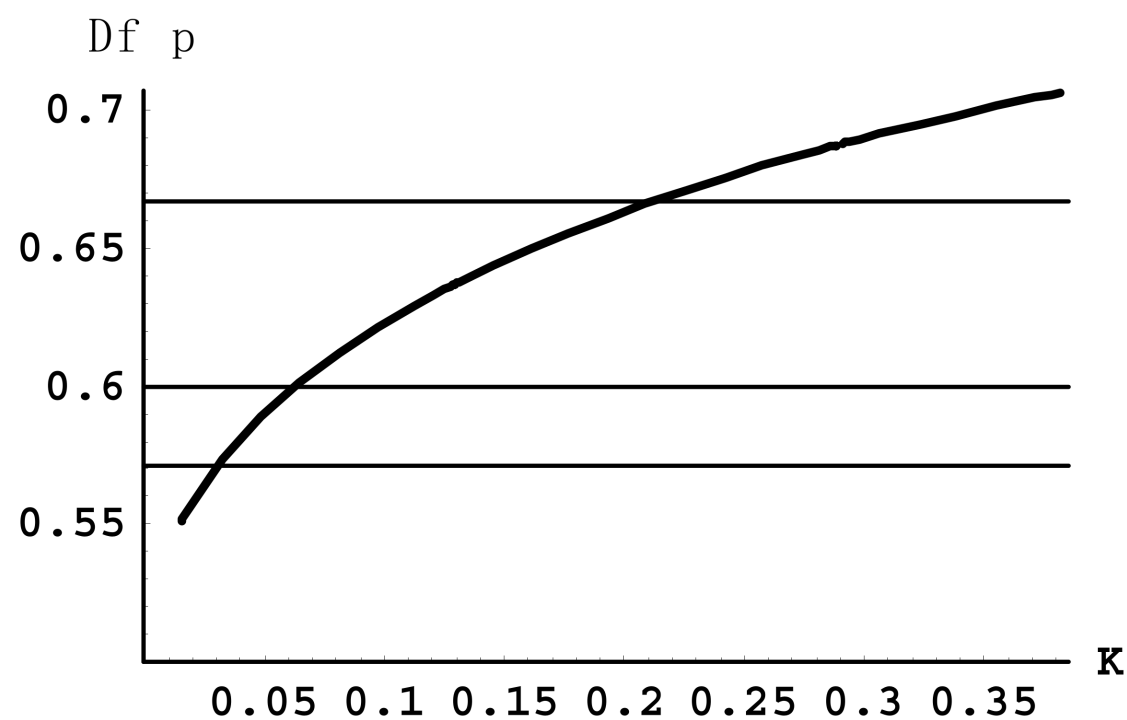

Figure 8. Intersections of the curve $\Delta \phi(\mathrm{K}) / \pi$ (thick line) with the tree horizontal lines corresponding to $2 / 3$ (the top thin line), $3 / 5$ (the middle thin line), and $4 / 7$ (the bottom thin line) [9].

For $n=3, m=5$, the angle $\phi$ completes 3 full circles during 5 periods of the $\theta$-oscillations. This occurs for $\mathrm{K}=0.0632$. The corresponding frequency of the $\theta$-oscillations is 1.2223 in units of $2 \mathrm{eD} /\left(\mathrm{mr}^{4}\right)$. In this case, the intensity of the spectral line emitted at the frequency 3.6669 in units of $2 \mathrm{eD} /\left(\mathrm{mr}^{4}\right)$, which is the triple-frequency of the $\theta$-oscillations, would be enhanced (compared to the intensity of the second harmonic of the frequency of the $\theta$-oscillations) due to the resonance with the fifth harmonic of the frequency of the $\varphi$-precession.

For $\mathrm{n}=4, \mathrm{~m}=7$, the angle $\phi$ completes 4 full circles during 7 periods of the $\theta$-oscillations. This occurs for $\mathrm{K}=0.0310$. The corresponding frequency of the $\theta$-oscillations is 1.2105 in units of $2 \mathrm{eD} /\left(\mathrm{mr}^{4}\right)$. In this case, the intensity of the spectral line emitted at the frequency 4.8420 in units of $2 \mathrm{eD} /\left(\mathrm{mr}^{4}\right)$, which is the quadruple-frequency of the $\theta$-oscillations, would be enhanced (compared to the intensity of the third harmonic of the frequency of the $\theta$-oscillations) due to the resonance with the seventh harmonic of the frequency of the $\varphi$-precession.

Observing the enhanced intensity of the spectral lines at the frequencies indicated above would be the manifestation of the states of the truly periodic motion of the electron around the polar molecule.

Thus, it is seen that the $\phi$-precessions can actually occur on the same time scale as the $\theta$-oscillations. It is true also in the general case. Therefore, the statement from [8] that "in general the motion should consist of large oscillations with respect to the polar angle $\theta$ combined with a slow precession about the z-axis" does not seem to be correct.

\section{Effects of a Magnetic Field of an Arbitrary Strength}

In this section, we consider the same system as in Section 2 but under a uniform magnetic field $\mathbf{B}$ along the $\mathbf{z}$-axis. Thus, the axial symmetry is preserved. The analytical results presented below were obtained for the arbitrary strength of the magnetic field.

Following paper [10], the Lagrangian can be written in the following form:

$$
\mathrm{L}=\mathrm{m}\left[(\mathrm{dr} / \mathrm{dt})^{2}+\mathrm{r}^{2}(\mathrm{~d} \theta / \mathrm{dt})^{2}+\mathrm{r}^{2} \sin ^{2} \theta(\mathrm{d} \varphi / \mathrm{dt})^{2}\right] / 2+\mathrm{eD} \cos \theta / \mathrm{r}^{2}-\left(\mathrm{mr}^{2} / 2\right)(\mathrm{d} \varphi / \mathrm{dt}) \Omega .
$$

In Equation (35):

$$
\Omega=\mathrm{eB} /(\mathrm{mc})
$$

is the scaled magnetic field: $\Omega>0$ or $\Omega<0$ if $\mathbf{B}$ is parallel or antiparallel to $\mathbf{D}$, respectively. 
The first equation of the motion is as follows:

$$
\mathrm{d}[\partial \mathrm{L} / \partial(\mathrm{d} \varphi / \mathrm{dt})] / \mathrm{dt}=\partial \mathrm{L} / \partial \varphi=0,
$$

so that:

$$
\partial \mathrm{L} / \partial(\mathrm{d} \varphi / \mathrm{dt})=\mathrm{mr}^{2}\left[(\mathrm{~d} \varphi / \mathrm{dt}) \sin ^{2} \theta-\Omega / 2\right]=\mathrm{p}_{\varphi}=\text { const. }
$$

From Equation (38), we find:

$$
\mathrm{d} \phi / \mathrm{dt}=\left[\Omega / 2+\mathrm{p}_{\phi} /\left(\mathrm{mr}^{2}\right)\right] / \sin ^{2} \theta
$$

so that:

$$
\phi(t)=\int \mathrm{dt}\left\{\Omega / 2+\mathrm{p}_{\varphi} /\left[\mathrm{mr}^{2}(\mathrm{t})\right]\right\} / \sin ^{2} \theta(\mathrm{t}) .
$$

Equation (40) allows determining the time evolution of the $\phi$-coordinate if the timeevolution of $r$ - and $\theta$-coordinates are known.

From the other Lagrange equations of the following motion:

$$
\mathrm{d}[\partial \mathrm{L} / \partial(\mathrm{dr} / \mathrm{dt})] / \mathrm{dt}=\partial \mathrm{L} / \partial \mathrm{r}
$$

we find:

$$
\mathrm{m}\left(\mathrm{d}^{2} \mathrm{r} / \mathrm{dt}^{2}\right)-\left[\mathrm{mr}(\mathrm{d} \theta / \mathrm{dt})^{2}+\operatorname{mrsin}^{2} \theta(\mathrm{d} \varphi / \mathrm{dt})^{2}-2 \mathrm{eD} \cos \theta / \mathrm{r}^{3}+\operatorname{mr} \Omega(\mathrm{d} \varphi / \mathrm{dt}) / 2\right]=0
$$

From the Lagrangian in Equation (35) follows the expression for the total energy E:

$$
E=m\left[(d r / d t)^{2}+r^{2}(d \theta / d t)^{2}+r^{2} \sin ^{2} \theta(d \varphi / d t)^{2}\right] / 2-e D \cos \theta / r^{2}+\left(m r^{2} / 2\right)(d \varphi / d t) \Omega .
$$

From the combination of Equations (42) and (43), the following relation can be obtained:

$$
2 E=m r\left(d^{2} r / d t^{2}\right)+m(d r / d t)^{2}=m d[r(d r / d t)] / d t .
$$

By integrating Equation (44), we obtain:

$$
\mathrm{r}(\mathrm{dr} / \mathrm{dt})=2 \mathrm{Et} / \mathrm{m}+\text { const. }
$$

From Equation (45), it is easy to find out that the bound motion is possible only for the energy $\mathrm{E}=0$ and const $=0$, leading to $\mathrm{dr} / \mathrm{dt}=0$, so that $\mathrm{r}=0$ : the motion on the sphere. Then, Equation (43) takes the following form:

$$
\mathrm{m}\left[(\mathrm{dr} / \mathrm{dt})^{2}+\mathrm{r}^{2}(\mathrm{~d} \theta / \mathrm{dt})^{2}+\mathrm{r}^{2} \sin ^{2} \theta(\mathrm{d} \phi / \mathrm{dt})^{2}\right] / 2-\mathrm{eD} \cos \theta / \mathrm{r}^{2}+\left(\mathrm{mr}^{2} / 2\right)(\mathrm{d} \phi / \mathrm{dt}) \Omega=0 .
$$

On substituting d $\phi / d t$ from Equation (39) in Equation (46), we obtain:

$(\mathrm{d} \theta / \mathrm{dt})^{2}+\mathrm{p}_{\phi}{ }^{2} /\left(\mathrm{m}^{2} \mathrm{r}^{4} \sin ^{2} \theta\right)+2 \mathrm{p}_{\phi} \Omega /\left(\mathrm{mr}^{2} \sin ^{2} \theta\right)+3 \Omega^{2} /\left(4 \sin ^{2} \theta\right)-2 \mathrm{eD} \cos \theta /\left(m r^{4}\right)=0$.

So, the two angular variables become separated.

After denoting $x=\cos \theta$, Equation (47) becomes:

$$
(\mathrm{dx} / \mathrm{dt})^{2}=\left[2 \mathrm{eD} /\left(\mathrm{mr}^{4}\right)\right] \mathrm{y}(\mathrm{x}, \mathrm{K}), \quad \mathrm{y}(\mathrm{x}, \mathrm{K})=\left(-\mathrm{x}^{3}+\mathrm{x}-\mathrm{K}\right),
$$

where:

$$
\mathrm{K}=\left[3 \mathrm{mr}^{4} /(8 \mathrm{eD})\right]\left[\Omega+2 \mathrm{p}_{\varphi} /\left(\mathrm{mr}^{2}\right)\right]\left[\Omega+2 \mathrm{p}_{\varphi} /\left(3 \mathrm{mr}^{2}\right)\right] .
$$

The polynomial $\mathrm{y}(\mathrm{x}, \mathrm{K})$ in Equation (48) has three roots with respect to $\mathrm{x}$, as follows:

$$
x_{1}(K)=\left\{-(-2)^{1 / 3}+\left[(-2)^{2 / 3} / 6\right]\left[\left(729 K^{2}-108\right)^{1 / 2}-27 K\right]^{2 / 3}\right\} /\left[\left(729 K^{2}-108\right)^{1 / 2}-27 K\right]^{1 / 3},
$$

$\mathrm{x}_{2}(\mathrm{~K})$ (presented in Equation (10)), and $\mathrm{x}_{3}(\mathrm{~K})$ (presented in Equation (11)). 
Obviously, the left side of the first formula in Equation (48) is non-negatively defined, so that the polynomial $y(x, K)$ should be positive or zero. On denoting the following:

$$
\mathrm{u}=\mathrm{mr}^{4} \Omega / \mathrm{p}_{\phi},
$$

Equation (49) becomes:

$$
\mathrm{K}=\left[\mathrm{p}_{\phi} /(2 \mathrm{meD})\right]\left(3 \mathrm{u}^{2} / 4+2 \mathrm{u}+1\right)
$$

From Equation (52), it is easy to find out that the parameter $\mathrm{K}$ can be negative for the values of $u$ from the interval $(-2,-2 / 3)$. (The negative values of $u$ correspond to the antiparallel configuration of vectors $\mathbf{B}$ and $\mathbf{D}$.) This is a new range of motion (with respect to the coordinate $\theta$ ) that was impossible at the zero field.

There are three cases for the $x$-motion (we remind the $x=\cos \theta$ ):

A. In the case of $0<\mathrm{K}<2 / 3^{3 / 2}$, the $x$-motion is in the segment $\left[\mathrm{x}_{2}(\mathrm{~K}), \mathrm{x}_{3}(\mathrm{~K})\right]$;

B. In the case of $-2 / 3^{3 / 2}<K<0$, the $x$-motion is either in the segment $\left[x_{2}(K), 1\right]$, or in the segment $\left[-1, \mathrm{x}_{1}(\mathrm{~K})\right]$. (Reminder: $-1<\mathrm{x}_{1}(\mathrm{~K})<1 / 3^{1 / 2}$ );

C. In the case of $K<-2 / 3^{3 / 2}$, the $x$-motion is in the segment $[-1,1]$.

Obviously, now there are additional ranges of the $x$-motion compared to the no-field situation where the $x$-motion was allowed only in the segment $\left[\mathrm{x}_{2}(\mathrm{~K}), \mathrm{x}_{3}(\mathrm{~K})\right]$.

Let us now obtain results for some particular cases.

1. $\mathrm{K}=\mathrm{K}_{\max }=2 / 3^{3 / 2}$. The electron rotates along the parallel of latitude, corresponding to $\theta=\arccos \left(1 / 3^{1 / 2}\right)$, with the following angular frequency:

$$
\mathrm{d} \phi / \mathrm{dt}=(3 / 4)\left[\Omega+2 \mathrm{p}_{\phi} /\left(\mathrm{mr}^{2}\right)\right]
$$

so that the period is:

$$
\mathrm{T}=(8 \pi / 3) /\left[\Omega+2 \mathrm{p}_{\phi} /\left(\mathrm{mr}^{2}\right)\right] .
$$

For $\mathrm{K}=2 / 3^{3 / 2}$, Equation (49) can be rewritten as follows:

$$
\left[\Omega+2 \mathrm{p}_{\phi} /\left(\mathrm{mr}^{2}\right)\right]\left[\Omega+2 \mathrm{p}_{\phi} /\left(3 \mathrm{mr}^{2}\right)\right]=16 \mathrm{eD} /\left(3^{5 / 2} \mathrm{mr}^{4}\right) .
$$

Using Equation (55), we can represent the period from Equation (20) in the following alternative form:

$$
\mathrm{T}=\left[3^{3 / 2} \pi \mathrm{mr}^{4} /(2 \mathrm{eD})\right]\left[\Omega+2 \mathrm{p}_{\phi} /\left(3 \mathrm{mr}^{2}\right)\right] .
$$

From Equation (55), it is seen that neither $\left[\Omega+2 \mathrm{p}_{\phi} /\left(\mathrm{mr}^{2}\right)\right]$ nor $\left[\Omega+2 \mathrm{p}_{\phi} /\left(3 \mathrm{mr} \mathrm{r}^{2}\right)\right]$ can vanish. So, from Equation (54), it follows that $\mathrm{T}$ cannot become infinite, and from Equation (56), it follows that $\mathrm{T}$ cannot vanish.

On introducing the following notations:

$$
\mathrm{w}=\mathrm{p}_{\phi} /(\mathrm{meD})^{1 / 2}, \quad \mathrm{~b}=3 \mathrm{mr}^{2} \Omega /(\mathrm{meD})^{1 / 2},
$$

Equation (55) takes the form:

$$
b^{2}+8 w b+12 w^{2}-16 / 3^{1 / 2}=0 .
$$

Equation (58) has the following two solutions:

$$
\mathrm{b}_{ \pm}=-4 \mathrm{w} \pm\left(4 \mathrm{w}^{2}+16 / 3^{1 / 2}\right)^{1 / 2}
$$

Equation (59) demonstrates that for any value of scaled projection w of the angular momentum, there exist two values of $b$ (i.e., of the scaled magnetic field according to Equation (57), for which the electron moves at the fixed value of $\theta=\arccos \left(1 / 3^{1 / 2}\right)$ along the parallel of latitude. This is a clear distinction from the zero field situation, where such motion could occur only for $\mathrm{w}= \pm 2 / 3^{3 / 4}= \pm 0.8774$. Moreover, there are two values of 
$\mathrm{b}=4 / 3^{1 / 4}=3.039$ and $\mathrm{b}=-4 / 3^{1 / 4}=-3.039$ (i.e., two corresponding values of $\mathrm{B}$ according to the definition of $b$ in Equation (57) enabling such electron motion even for $p_{\phi}=0$ ).

2. $K=0$. From Equation (49), it follows that $K=0$ for the following two values of the scaled (to the frequency dimension) magnetic field:

$$
\Omega_{1}=-2 \mathrm{p}_{\phi} /\left(m r^{2}\right), \quad \Omega_{2}=-2 \mathrm{p}_{\phi} /\left(3 \mathrm{mr}^{2}\right) .
$$

Here, the most interesting situation is the one where $\Omega=\Omega_{1}$. The electron moves in a semicircular path in a meridional plane in the northern hemisphere for any $\mathrm{p}_{\phi}$, while in the no-field case, this trajectory was valid only for the zero value of $\mathrm{p}_{\phi}$.

For $K=0$, in Section 2, the explicit dependence of the scaled time $\tau$ on $x=\cos \theta$ was obtained, given by Equation (18). For the range $0<x<1$ where the motion occurs, the right side of Equation (18) yields real values. We remind the reader that for the subcase of $x<<1$, Equation (18) is simplified to Equation (19).

Now, we provide some further details (in addition to those given by Equations (22), (26) and (29)) for the general situation where $\mathrm{K}$ is arbitrary. Figure 9 displays the angular variable $\phi$ versus $\mathrm{x}$ (during the half-period of the $\mathrm{x}$-motion) for $\mathrm{K}=0.15$ (solid line) and $\mathrm{K}=0.3$ (dashed line).

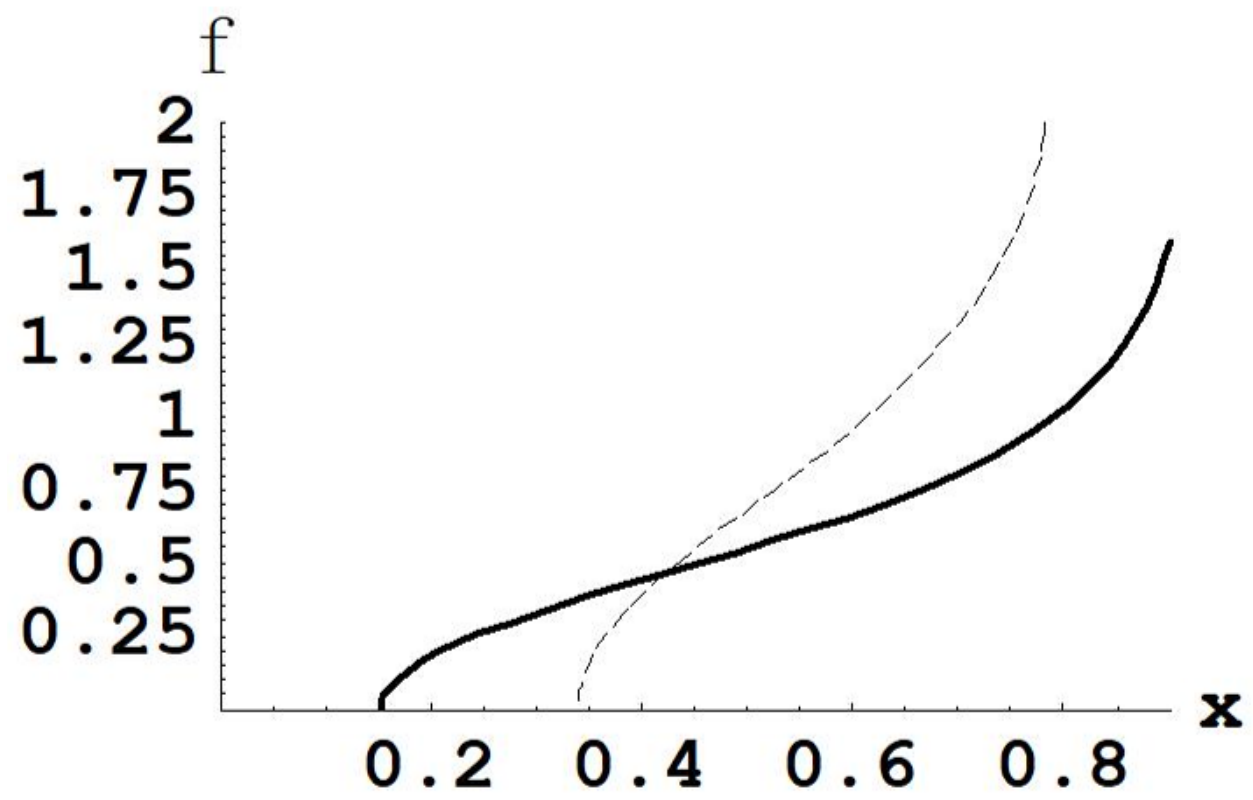

Figure 9. Angular variable $\phi$ versus $x$ (during the half-period of the $\mathrm{x}$-motion) for $\mathrm{K}=0.15$ (solid line) and $K=0.3$ (dashed line).

Table 1 presents the scaled period $\mathrm{T}_{\theta}$ (in units of $\mathrm{mr}^{4} /(2 \mathrm{eD})$ ) of the $\mathrm{x}$-motion versus the parameter $\mathrm{K}$ in the negative range of $\mathrm{K}$. It is a counterintuitive result that the period of the $\mathrm{x}$-motion depends on $\mathrm{K}$ non-monotonically.

Table 1. Scaled period $\mathrm{T}_{\theta}$ of the $\theta$-motion in units of $\mathrm{mr}^{4} /(2 \mathrm{eD})$ versus parameter $\mathrm{K}$ for the range of negative values of $\mathrm{K}$.

\begin{tabular}{ccccccc}
\hline $\mathrm{K}$ & -0.35 & -0.25 & -0.15 & -0.1 & -0.05 & 0 \\
\hline $\mathrm{T}_{\theta}$ & 6.07 & 5.17 & 4.91 & 4.88 & 4.91 & 5.24 \\
\hline
\end{tabular}

Figure 10 displays the angular variable $\phi$ versus $x$ (during the half-period of the $\mathrm{x}$-motion) for $\mathrm{K}=-0.15$ (solid line) and $\mathrm{K}=-0.3$ (dashed line). 


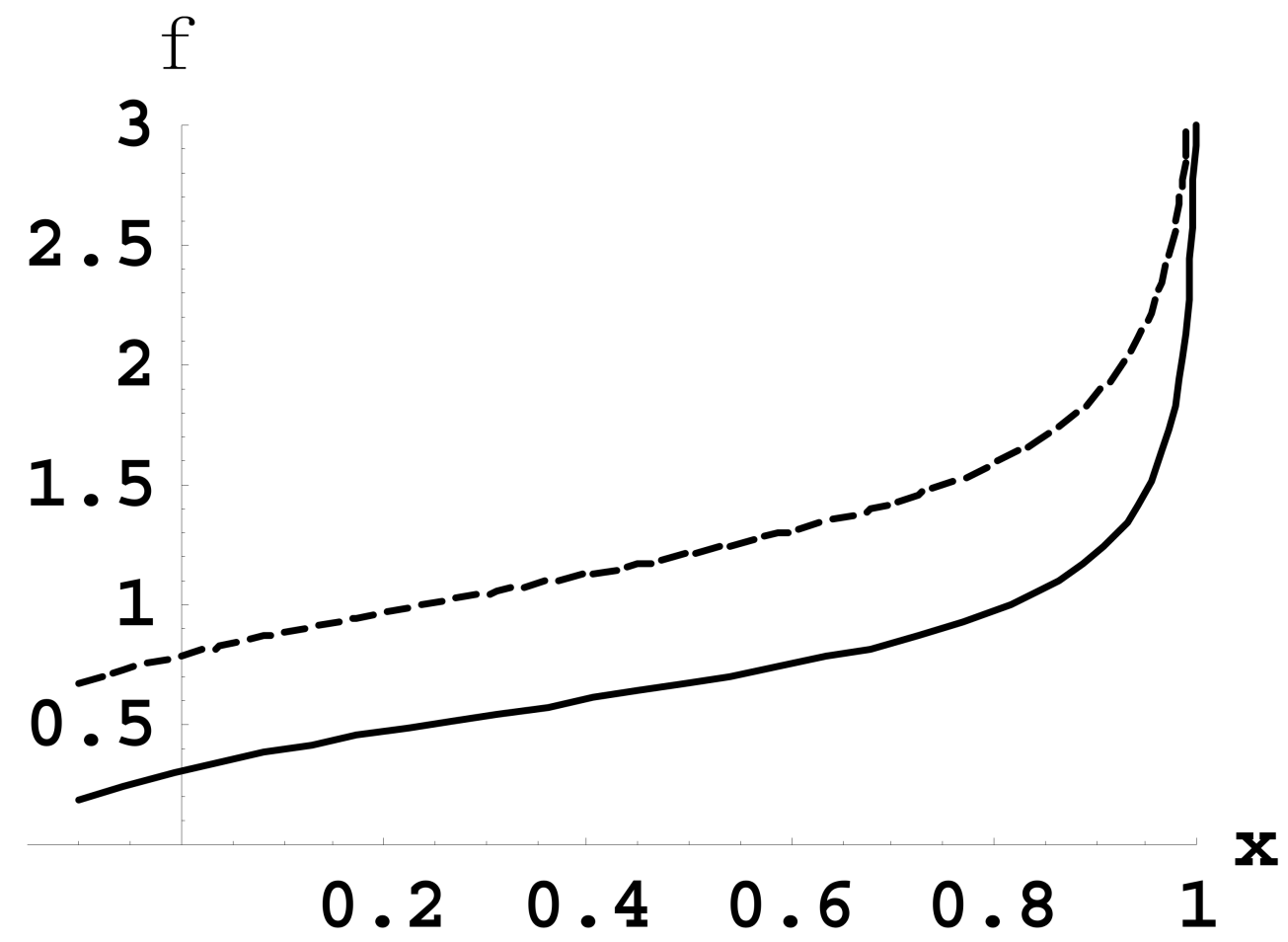

Figure 10. Angular variable $\phi$ versus $x$ (during the half-period of the $x$-motion) for $K=-0.15$ (solid line) and $\mathrm{K}=-0.3$ (dashed line).

Further details on the effects of the magnetic field on this system can be found in paper [10].

\section{Effects of an Electric Field of an Arbitrary Strength}

In this Section we consider the same system as in Section 2, but under a uniform magnetic field $\mathbf{F}$ parallel or antiparallel to the dipole. Thus, again the axial symmetry is preserved. The analytical results presented below are obtained for the arbitrary strength of the electric field.

The Lagrangian has the form

$$
\mathrm{L}=\mathrm{m}\left[(\mathrm{dr} / \mathrm{dt})^{2}+\mathrm{r}^{2}(\mathrm{~d} \theta / \mathrm{dt})^{2}+\mathrm{r}^{2} \sin ^{2} \theta(\mathrm{d} \phi / \mathrm{dt})^{2}\right] / 2+\mathrm{eD} \cos \theta / \mathrm{r}^{2}-\mathrm{eFr} \cos \theta,
$$

where $m$ and e are the mass and the absolute value of the electron charge, respectively. The quantity $\mathrm{F}$ can be positive or negative depending on whether the electric field is parallel or antiparallel to the dipole.

From one of the Lagrange equations of the following motion:

$$
\mathrm{d}[\partial \mathrm{L} / \partial(\mathrm{d} \phi / \mathrm{dt})] / \mathrm{dt}=\partial \mathrm{L} / \partial \phi=0
$$

it follows that

$$
\partial \mathrm{L} / \partial(\mathrm{d} \phi / \mathrm{dt})=\mathrm{mr}^{2} \sin ^{2} \theta(\mathrm{d} \phi / \mathrm{dt})=\mathrm{p}_{\phi}=\text { const },
$$

where $\mathrm{p}_{\phi}$ is the projection of the angular momentum on the dipole

From Equation (63), we find the following:

$$
\mathrm{d} \varphi / \mathrm{dt}=\mathrm{p}_{\phi} /\left(m r^{2} \sin ^{2} \theta\right) .
$$

so that:

$$
\phi(t)=\int \mathrm{dt} \mathrm{p}_{\phi} /\left[\mathrm{mr}^{2}(\mathrm{t}) \sin ^{2} \theta(\mathrm{t})\right] .
$$


From Equation (65), one can determine the time evolution of the $\phi$-coordinate if the time-evolution of the $r$ - and $\theta$-coordinates are known.

From the other Lagrange equations of the motion

$$
\mathrm{d}[\partial \mathrm{L} / \partial(\mathrm{dr} / \mathrm{dt})] / \mathrm{dt}=\partial \mathrm{L} / \partial \mathrm{r}
$$

we obtain:

$$
\mathrm{m}\left(\mathrm{d}^{2} \mathrm{r} / \mathrm{dt} \mathrm{t}^{2}\right)-\left[\mathrm{mr}(\mathrm{d} \theta / \mathrm{dt})^{2}+\mathrm{mrsin}^{2} \theta(\mathrm{d} \phi / \mathrm{dt})^{2}-2 \mathrm{eD} \cos \theta / \mathrm{r}^{3}-\mathrm{eFr} \cos \theta\right]=0 .
$$

The formula for the total energy E, following from the Lagrangian in Equation (61), is:

$$
\mathrm{E}=\mathrm{m}\left[(\mathrm{dr} / \mathrm{dt})^{2}+\mathrm{r}^{2}(\mathrm{~d} \theta / \mathrm{dt})^{2}+\mathrm{r}^{2} \sin ^{2} \theta(\mathrm{d} \phi / \mathrm{dt})^{2}\right] / 2+\left(\mathrm{eFr}-\mathrm{eD} / \mathrm{r}^{2}\right) \cos \theta .
$$

Now, we multiply both sides of Equation (67) by $r$ and add to it Equation (68) multiplied by 2 to obtain the following:

$$
2 \mathrm{E}=\mathrm{mr}\left(\mathrm{d}^{2} \mathrm{r} / \mathrm{dt}^{2}\right)+\mathrm{m}(\mathrm{dr} / \mathrm{dt})^{2}+3 \mathrm{eFr} \cos \theta=\mathrm{md}[\mathrm{r}(\mathrm{dr} / \mathrm{dt})] / \mathrm{dt}+3 \mathrm{eFr} \cos \theta,
$$

so that:

$$
\mathrm{d}[\mathrm{r}(\mathrm{dr} / \mathrm{dt})] / \mathrm{dt}=2 \mathrm{E} / \mathrm{m}-(3 \mathrm{eFr} / \mathrm{m}) \cos \theta .
$$

From the last remaining Lagrange equations of the motion

$$
\mathrm{d}[\partial \mathrm{L} / \partial(\mathrm{d} \theta / \mathrm{dt})] / \mathrm{dt}=\partial \mathrm{L} / \partial \theta
$$

we find the following:

$$
m r^{2}\left(d^{2} \theta / d t^{2}\right)+2 m r(d r / d t)(d \theta / d t)=m r^{2} \sin \theta \cos \theta(d \phi / d t)^{2}+\left(e F r-e D / r^{2}\right) \sin \theta .
$$

After substituting $\mathrm{d} \phi / \mathrm{dt}$ from Equation (64) in Equation (72), the latter equation would depend only on $r$ and $\theta$. Together with Equation (70), it would constitute the system of two equations for the two unknowns ( $\mathrm{r}$ and $\theta$ ) in the general case.

For obtaining explicit analytical results, from now on we consider the situation where the electron revolves around the dipole in a circular orbit (concentric with the dipole) perpendicular to the dipole axis, so that $r=$ const and $\theta=$ const. Then, from Equation (70) follows the interrelation between these two constant values:

$$
\cos \theta=2 \mathrm{E} /(3 \mathrm{eFr}) .
$$

From Equation (64), it follows that:

$$
\mathrm{d} \phi / \mathrm{dt}=\mathrm{p}_{\phi} /\left(\mathrm{mr}^{2} \sin ^{2} \theta\right)=\text { const }=\omega,
$$

where the constant angular velocity is denoted by $\omega$. From Equation (67) one obtains:

$$
\mathrm{mr} \omega^{2} \sin ^{2} \theta-\left(2 \mathrm{eD} / \mathrm{r}^{3}+\mathrm{eFr}\right) \cos \theta=0 .
$$

From Equation (72) we obtain:

$$
\left[\mathrm{mr}^{2} \omega^{2} \cos \theta+\mathrm{eFr}-\mathrm{eD} / \mathrm{r}^{2}\right] \sin \theta=0 .
$$

Since $\sin \theta=0$ (i.e., $\theta=0$ or $\theta=\pi$ ) is the unphysical solution, then from Equation (76) follows:

$$
\mathrm{mr}^{2} \omega^{2} \cos \theta+\mathrm{eFr}-\mathrm{eD} / \mathrm{r}^{2}=0 .
$$

On substituting $\cos \theta$ from Equation (73) in Equation (77), we find:

$$
\omega^{2}=\left[3 e^{2} F /(2 m E)\right]\left(D / r^{3}-F\right) .
$$


On substituting $\cos \theta$ from Equation (73) in Equation (76), we obtain:

$$
\omega^{2}=[2 \mathrm{E} /(3 \mathrm{mF})]\left(2 \mathrm{D} / \mathrm{r}^{3}+\mathrm{F}\right) /\left[\mathrm{r}^{2}-4 \mathrm{E}^{2} /\left(9 \mathrm{e}^{2} \mathrm{~F}^{2}\right)\right] .
$$

After equating to each other the right sides of Equations (78) and (79) and doing simple transformations, we obtain the following equation containing only one unknown quantity-the quantity $r$

$$
r^{5}-b r^{2}+3 a b=0,
$$

where we introduced the following notations:

$$
\mathrm{a}=4 \mathrm{E}^{2} /\left(9 \mathrm{e}^{2} \mathrm{~F}^{2}\right) \quad \mathrm{b}=\mathrm{D} / \mathrm{F} .
$$

It is important to note that while the quantity a from Equation (81) is always nonnegative, the quantity $\mathrm{b}$ can be positive (if $\mathrm{F}>0$ ) or negative (if $\mathrm{F}<0$ ). Equation (80) can be also represented as follows:

$$
r^{5}-b r^{2}+4 \operatorname{sign}(b)|b|^{5 / 3} E_{s}{ }^{2} / 3=0,
$$

where we denoted the following:

$$
E_{\mathrm{S}}=|\mathrm{E}| /\left(\mathrm{eD}^{1 / 3}|\mathrm{~F}|^{2 / 3}\right) .
$$

Physically, $\mathrm{E}_{\mathrm{s}}$ is the scaled dimensionless absolute value of the energy. While in Equation (80), the left side has a slightly simpler form than in Equation (82), the latter equation explicitly contains the scaled dimensionless absolute value of the energy $\mathrm{E}_{\mathrm{s}}$, which turns out to be the physical parameter controlling (together with the parameter $b$ ) various solutions of Equation (82), as will be shown below.

We seek the solution of Equation (82) in the following form:

$$
\mathrm{r}=\mathrm{c}|\mathrm{b}|^{1 / 3}, \quad \mathrm{c}=\text { const }>0 .
$$

On substituting Equation (84) in Equation (82), we obtain:

$$
\mathrm{P}(\mathrm{c})=\mathrm{c}^{5}-\mathrm{c}^{2} \operatorname{sign}(\mathrm{b})+(4 / 3) \mathrm{E}_{\mathrm{s}}^{2} \operatorname{sign}(\mathrm{b})=0 .
$$

Let us first consider the situation where $\mathrm{b}>0$, i.e., $\mathrm{F}>0$, meaning that the electric field points in the same direction as the dipole. Then, Equation (85) takes the following form:

$$
\mathrm{P}(\mathrm{c})=\mathrm{c}^{5}-\mathrm{c}^{2}+4 \mathrm{E}_{\mathrm{s}}^{2} / 3=0 .
$$

According to one of the theorems of algebra-namely, Descartes's rule of signs-for $\mathrm{b}>0$, the polynomial $\mathrm{P}(\mathrm{c})$ from Equation (86) has either two or zero positive roots (we are not interested in negative roots-they are unphysical since it should be $r>0$ ). By equating the derivative of $\mathrm{P}(\mathrm{c})$ to zero, it is easy to find after a simple analysis that at $\mathrm{c}=0$ the polynomial has a local maximum $\mathrm{P}_{\max }=(4 / 3) \mathrm{E}_{\mathrm{s}}{ }^{2}$ and that it has the absolute minimum at $\mathrm{c}=(2 / 5)^{1 / 3}$ :

$$
\mathrm{P}_{\min }=(2 / 5)^{5 / 3}-(2 / 5)^{2 / 3}+4 \mathrm{E}_{\mathrm{s}}^{2} / 3=4 \mathrm{E}_{\mathrm{s}}^{2} / 3-0.326 \text {. }
$$

Obviously, for the existence of two different positive roots, there should be $P_{\min }<0$, leading to the following requirement:

$$
\mathrm{E}_{\mathrm{S}}<\mathrm{E}_{\mathrm{s}, \max }=0.494 .
$$

At $E_{S}<E_{S, \max }$, the two positive roots merge together at $c=(2 / 5)^{1 / 3}=0.737$-see Figure 11. 


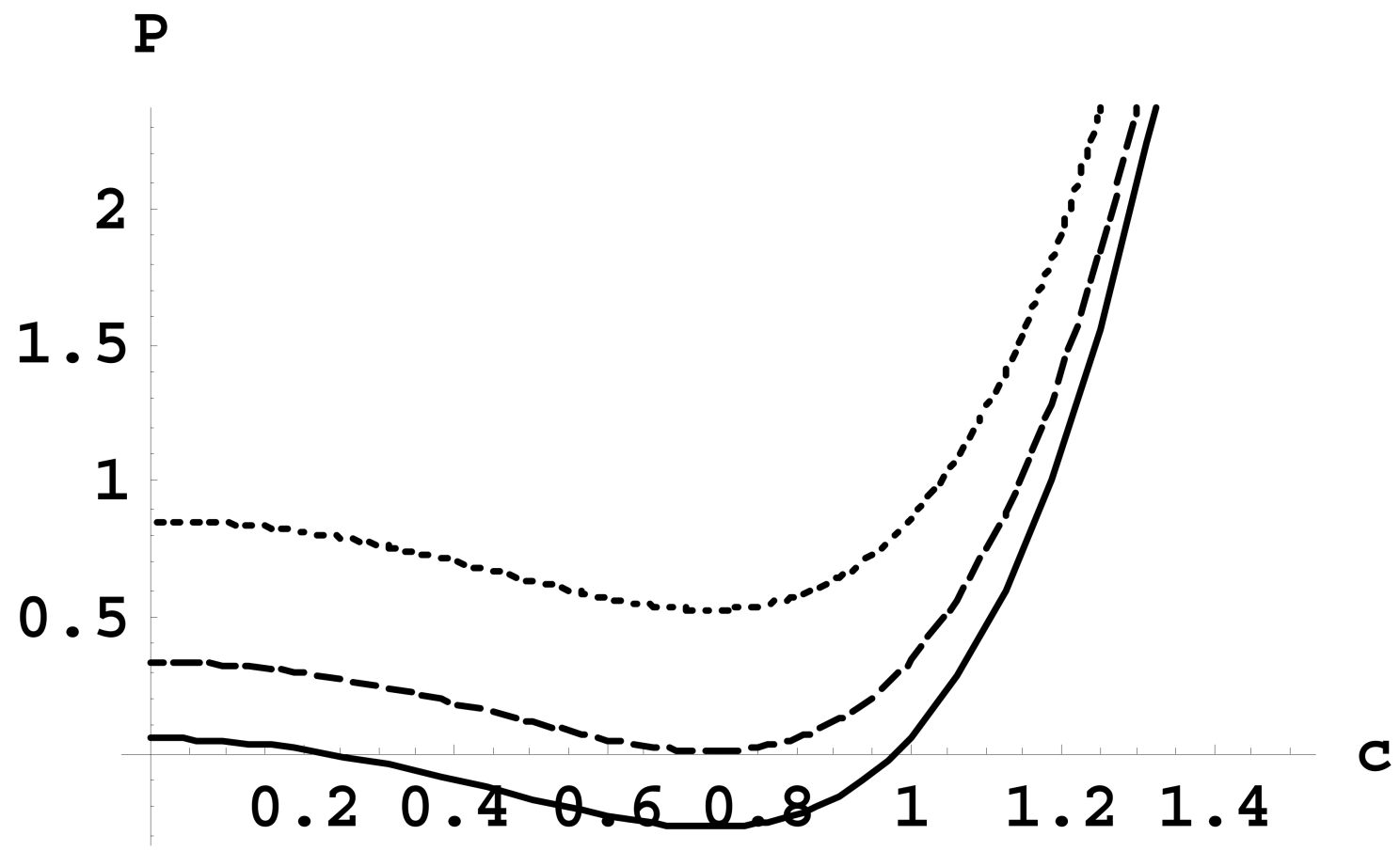

Figure 11. Plot of the polynomial $\mathrm{P}(\mathrm{c})$ from Equation (86) for the three values of the scaled dimensionless absolute value of the energy $\mathrm{E}_{\mathrm{s}}$ : 0.2 (solid line), 0.5 (dashed line), and 0.8 (dotted line).

It is well known that there are no exact analytical solutions for the roots of polynomials of the 5th (or higher) degree. However, we can find approximate analytical solutions for the case where $E_{\mathrm{S}}<<1$, as follows.

One of the positive roots corresponds to the situation where in Equation (86) the first term is much smaller than the other two terms. The truncated equation has the following form:

$$
-\mathrm{c}^{2}+4 \mathrm{E}_{\mathrm{s}}^{2} / 3 \approx 0
$$

yielding the root:

$$
\mathrm{c}_{1} \approx 2 \mathrm{E}_{\mathrm{s}} / 3^{1 /}
$$

so that:

$$
\mathrm{r}_{1} \approx 2 \mathrm{E}_{\mathrm{s}}|\mathrm{b}|^{1 / 3} / 3^{1 / 2}=2|\mathrm{E}| /\left(3^{1 / 2} \mathrm{e}|\mathrm{F}| .\right.
$$

It is easy to verify that at $r=r_{1}$, the first term in Equation (86) is indeed much smaller than the two other terms, given that $\mathrm{E}_{\mathrm{S}}<<1$.

On substituting $r_{1}$ from Equation (91) in Equation (78), we find the following expression for the square of the electron revolution frequency (we remind that we still consider the situation where $\mathrm{F}>0$ ):

$$
\omega_{1}{ }^{2}=\left[3 \mathrm{e}^{2} \mathrm{~F} /(2 \mathrm{mE})\right]\left(\mathrm{D} / \mathrm{r}_{1}{ }^{3}-\mathrm{F}\right) \approx\left[3^{5 / 2} \mathrm{DF}^{4} /\left(16 \mathrm{mE}^{4}\right)\right] \operatorname{sign}(\mathrm{E}) .
$$

Obviously, $\omega_{1}^{2}$ should be positive. Consequently, this solution has the physical meaning only for $E>0$. Then, by substituting $r_{1}$ from Equation (91) in Equation (73), we obtain the corresponding value of $\cos \theta$ :

$$
\cos \theta_{1}=2 \mathrm{E} /(3 \mathrm{eFr}) \approx\left(1 / 3^{1 / 2}\right) \operatorname{sign}(\mathrm{E})=1 / 3^{1 / 2} .
$$

The second positive root corresponds to the situation where, in Equation (86), the third term is much smaller than the two other terms. The truncated equation has the form

$$
c^{5}-c^{2} \approx 0,
$$


yielding $c_{2} \approx 1$. It is seen that the third term in Equation (86) is indeed much smaller than the two other terms since $E_{S}<<1$. For obtaining a non-vanishing result for the square of the electron revolution frequency, one has to go to the next approximation. By substituting $c=(1+\alpha)$, where $|\alpha|<<1$, in Equation (86), we find the following:

$$
\mathrm{c}_{2} \approx 1-4 \mathrm{E}_{\mathrm{s}}^{2} / 9,
$$

so that:

$$
\mathrm{r}_{2} \approx\left(1-4 \mathrm{E}_{\mathrm{s}}^{2} / 9\right)(\mathrm{D} / \mathrm{F})^{1 / 3} .
$$

On substituting $\mathrm{r}_{2}$ from Equation (96) in Equation (78), we obtain:

$$
\omega_{2}^{2} \approx 2 \mathrm{EF}^{2 / 3} /\left(\mathrm{mD}^{2 / 3}\right) .
$$

Since $\omega_{2}^{2}$ should be positive, this second solution also has the physical meaning only for $E>0$. Then, by substituting $r_{2}$ from Equation (96) in Equation (73), we obtain the corresponding value of $\cos \theta$ :

$$
\cos \theta_{2} \approx(2 / 3) \mathrm{E}_{\mathrm{s}} \operatorname{sign}(\mathrm{E})=2 \mathrm{E}_{\mathrm{s}} / 3=2 \mathrm{E} /\left(\mathrm{eD}^{1 / 3} \mathrm{~F}^{2 / 3}\right)
$$

Now, we proceed to the situation where $\underline{b}<0$, i.e., $\mathrm{F}<0$. Then, Equation (85) takes the following form:

$$
\mathrm{P}(\mathrm{c})=\mathrm{c}^{5}+\mathrm{c}^{2}-4 \mathrm{E}_{\mathrm{s}}^{2} / 3=0 .
$$

According to Descartes's rule of signs, this equation has one and only one positive root for any $E_{\mathrm{s}}$. This is illustrated in Figure 12.

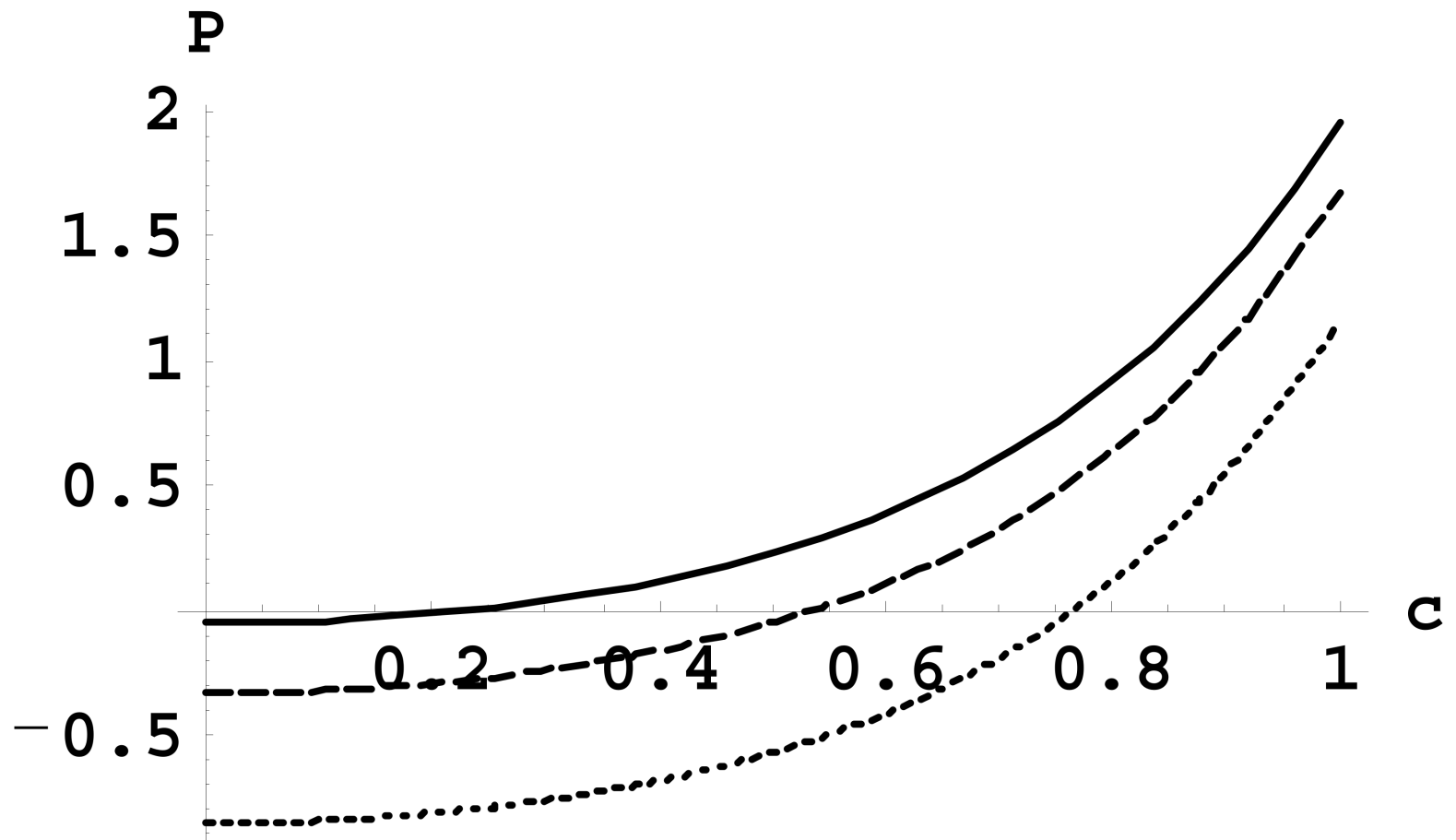

Figure 12. Plot of the polynomial $\mathrm{P}(\mathrm{c})$ from Equation (99) for the three values of the scaled dimensionless absolute value of the energy $\mathrm{E}_{\mathrm{s}}$ : 0.2 (solid line), 0.5 (dashed line), and 0.8 (dotted line).

For $E_{S}>>1$ or $E_{s}<<1$, we can find an approximate analytical result for the root of Equation (99), as follows. We start from the case of $E_{S}>>1$. In this case, the second term 
in Equation (99) is much smaller than the two other terms (see the proof below). The truncated equation has the following form:

$$
c^{5}-4 \mathrm{E}_{\mathrm{s}}^{2} / 3 \approx 0,
$$

yielding:

$$
\mathrm{c}_{3} \approx\left(4 \mathrm{E}_{\mathrm{s}}^{2} / 3\right)^{1 / 5} \text {. }
$$

For this value of $c_{3}$, the ratio of the second term in Equation (99) to, e.g., the first term is $1 / \mathrm{c}_{3}{ }^{3} \sim 1 / \mathrm{E}_{\mathrm{s}}{ }^{6 / 5}<<1$, so that the neglect of the second term is justified.

With the $c_{3}$ from Equation (101), we obtain:

$$
\mathrm{r}_{3} \approx\left(4 \mathrm{E}_{\mathrm{s}}{ }^{2} / 3\right)^{1 / 5}(\mathrm{D} /|\mathrm{F}|)^{1 / 3}=(2|\mathrm{E}|)^{2 / 5} \mathrm{D}^{1 / 5} /\left(3^{1 / 5} \mathrm{e}^{2 / 5}|\mathrm{~F}|^{3 / 5}\right) .
$$

On substituting $r_{3}$ from Equation (102) in Equation (73), we obtain:

$$
\cos \theta_{3} \approx-\left(2^{3 / 5} / 3^{4 / 5}\right) \mathrm{E}_{\mathrm{s}}{ }^{3 / 5} \operatorname{sign}(\mathrm{E}) .
$$

Obviously, in Equation (103) $\left|\cos \theta_{3}\right|>>1$ (since, in this this case, $E_{\mathrm{S}}>>1$ ), so that this formal mathematical solution does not have a physical meaning.

The last remaining case is $E_{S}<<1$ (while $F<0$ ). In this case, the first term in Equation (99) is much smaller than the other two terms (see the proof below). The truncated equation has the following form:

$$
c^{2}-4 \mathrm{E}_{\mathrm{s}}^{2} / 3 \approx 0,
$$

yielding:

$$
\mathrm{c}_{4} \approx 2 \mathrm{E}_{\mathrm{s}} / 3^{1 / 2},
$$

so that:

$$
\mathrm{r}_{4} \approx 2 \mathrm{E}_{\mathrm{s}}|\mathrm{b}|^{1 / 3} / 3^{1 / 2}=2|\mathrm{E}| /\left(3^{1 / 2} \mathrm{e}|\mathrm{F}|\right) .
$$

(The values of $c_{4}$ and $r_{4}$ coincide with the values of $c_{1}$ and $r_{1}$, respectively). It is again easy to verify that at $r=r_{4}$, the first term in Equation (99) is indeed much smaller than the two other terms, given that $\mathrm{E}_{\mathrm{s}}<<1$.

The expression for the square of the electron revolution frequency $\omega_{4}^{2}$ in this case differs by the sign from $\omega_{1}^{2}$ in Equation (92) because now $\mathrm{F}<0$ :

$$
\omega_{4}{ }^{2}=\left[3 \mathrm{e}^{2} \mathrm{~F} /(2 \mathrm{mE})\right]\left(\mathrm{D} / \mathrm{r}_{4}{ }^{3}-\mathrm{F}\right) \approx-\left[3^{5 / 2} \mathrm{DF}^{4} /\left(16 \mathrm{mE}^{4}\right)\right] \operatorname{sign}(\mathrm{E}) .
$$

Since $\omega_{4}^{2}$ should be positive, this solution has the physical meaning only for $\mathrm{E}<0$. Then, by substituting $\mathrm{r}_{4}$ from Equation (106) in Equation (73), we obtain the corresponding value of $\cos \theta$ :

$$
\cos \theta_{4}=2 \mathrm{E} /(3 \mathrm{eFr}) \approx-\left(1 / 3^{1 / 2}\right) \operatorname{sign}(\mathrm{E})=1 / 3^{1 / 2} .
$$

It should be emphasized that the results given by Equations (106)-(108) for $\mathrm{F}<0$ are quite remarkable. They correspond to the bound states of negative energies $(E<0)$. We remind readers that in the absence of the electric field (the situation studied in papers [6-9]), as well as in the presence of the magnetic field along the electric dipole (the situation presented in Section 3 of the current review), the only possible bound states were of zero energy. Thus, the electric field can stabilize the system into bound states of negative energies.

This is a counterintuitive result. Indeed, while a magnetic field typically inhibits the ionization of atomic systems, the electric field promotes the ionization and is thus considered as a destabilizing factor. However, the solution presented by Equations (106)-(108) for $\mathrm{F}<0$ (i.e., for the electric field antiparallel to the dipole) demonstrates that the electric field can work as the stabilizing factor for the Rydberg electron in the field of a point-like electric dipole. 
We note that the frequency of the electron revolution found above would manifest as the frequency of spectral lines emitted by the system. By measuring the frequency of the spectral lines and knowing the electric dipole moment D and the field strength $\mathrm{F}$, one can deduce the energy E-see, e.g., Equation (107).

\section{Conclusions}

In Section 2 of this review, we followed paper [9] to present the analysis of the classical bound motion of a Rydberg electron around a polar molecule. It was demonstrated that, generally, both the $\theta$-oscillations and the $\phi$-precessions can actually occur on the same time scale, so that the statement to the contrary from work [8] seems to be incorrect.

Furthermore, the relation between the dynamical variable $\phi$ and the dynamical variable $\theta$ was derived in the form of a one-fold integral in the general situation. In addition, an explicit analytical result for $\phi(\theta)$ was obtained for the situation where the parameter $\mathrm{K}$, which is the scaled square of the projection $p_{\phi}$ of the angular momentum on the axis of the electric dipole, is relatively small.

For the case of $K=0$, corresponding to the motion of the electron along a meridional semicircle crossing the North pole, an explicit analytical result for the dependence of time $t$ on $\theta$ was obtained. For the case of $\mathrm{K}=\mathrm{K}_{\max }=2 / 3^{3 / 2}$, where the electron goes in a circle, the circle being the parallel of latitude corresponding to $\theta=0.9553 \mathrm{rad}=54.74$ degrees, an explicit analytical result was derived for the period of the revolution.

Furthermore, in the general case, the time evolution of the dynamical variable $\theta$ and the period $\mathrm{T}_{\theta}$ of the $\theta$-oscillations was derived in the form of a one-fold integral. This was complemented by the corresponding explicit analytical expressions for the case of $\mathrm{K}<<1$.

In addition, in the general case, the change of the angular variable $\varphi$ during one half-period of the $\theta$-motion was derived in the form of a one-fold integral.

Finally, it was shown in detail at what values of the parameter $\mathrm{K}$, the conditionally periodic motion of the electron would become truly periodic. A general condition for the truly periodic motion of the electron was obtained and three examples were provided of the corresponding values of the parameter $\mathrm{K}$.

From the fundamental point of view, deriving all of the above analytical results was possible due to the axial symmetry of the system.

In Section 3, the same system from Section 2 was analyzed under a magnetic field parallel or antiparallel to the electric dipole, so that the axial symmetry was preserved. The analytical results were derived in paper [10] for the arbitrary strength of the magnetic field.

It was demonstrated that under the magnetic field, new ranges of the bound motion of the Rydberg electron open up. Especially important is that, as the magnetic field opened up several new ranges of the parameter $K$, in the range $0<\mathrm{K}<2 / 3^{3 / 2}$, the period of the $\theta$-oscillations has the non-monotonic dependence on $\mathrm{K}$. This is a counterintuitive result.

Next, while at $B=0$, the oscillations of the electron along a semicircle located in a meridional plane in the Northern hemisphere were possible only for the zero projection of the angular momentum $\mathrm{p}_{\phi}$ on the electric dipole; the magnetic field makes this motion possible for any value of $\mathrm{p}_{\phi}-$ provided that $\Omega=-2 \mathrm{p}_{\varphi} /\left(\mathrm{mr}^{2}\right)$, where $\Omega=\mathrm{eB} /(\mathrm{mc})$ is the scaled magnetic field.

In addition, while at $B=0$, the circular trajectory of the electron along the parallel of latitude at $\cos \theta=1 / 3^{1 / 2}$ was possible only for two values of the projection of the angular momentum corresponding to $\mathrm{p}_{\phi} /(\mathrm{meD})^{1 / 2}= \pm 2 / 3^{3 / 4}= \pm 0.8774$, a proper choice of the magnetic field strength enables this kind of motion for any value of $p_{\phi}$-see Equation (59).

In Section 4, the same system from Section 2 was analyzed under an electric field $\mathbf{F}$ parallel or antiparallel to the electric dipole, so that the axial symmetry was preserved. The analytical results were derived for the arbitrary strength of the electric field.

First, some results were obtained for the general case. Then, the focus was on the circular orbits of the electron, the orbits being concentric with the dipole axis and the orbital plane being perpendicular to the dipole axis. Analytical results were obtained for the revolution frequency, as well as for the polar angle and the radius of the orbit-for 
three different equilibrium states of the system: two states of a positive energy (i.e., when the electric field and the dipole are parallel) and one state of the negative energy (i.e., when the electric field and the dipole are antiparallel).

The existence of the equilibrium state of the negative energy is a counterintuitive result. Indeed, at $F=0$, the equilibrium state was possible only for the zero energy. This result is counterintuitive because it defies the usual expectation is that an electric field promotes the ionization: In the above situation, the electric field was found to be the stabilizing factor.

By observing the spectral lines emitted by the system and measuring their frequency, it is possible to deduce the energy by using our analytical results for the electron revolution frequency.

All of the above classical analytical results, obtained for the arbitrary strength of the magnetic and electric fields, provide a physical insight in the complicated dynamics of a Rydberg electron around a polar molecule. These analytical results, being valid for the arbitrarily large strength of the magnetic and electric fields, cannot be obtained analytically by the perturbation theory. The statement that the analytical results are obtained for the arbitrary strength of the magnetic and electric fields refers to the system "an electron plus an electric dipole". When the dipole is represented by a polar molecule, the following clarification is in order. The external electric field induces an additional dipole moment in the molecule. In this case, in the corresponding formulas from Section 4, the dipole moment $\mathrm{D}$ should include the contributions from both the built-in dipole moment and the induced one.

Funding: This work received no external funding.

Data Availability Statement: All data is included in the paper.

Conflicts of Interest: The author declares no conflict of interest.

\section{References}

1. Fermi, E.; Teller, E. The capture of negative mesotrons in matter. Phys. Rev. 1947, 72, 399-408. [CrossRef]

2. Connolly, K.; Griffiths, D.J. Critical dipoles in one, two, and three dimensions. Am. J. Phys. 2007, 75, 524-531. [CrossRef]

3. Turner, J.E.; Fox, K. Classical motion of an electron in an electric-dipole field. I. Finite dipole case. J. Phys. A 1968, 1, 118-123. [CrossRef]

4. Kryukov, N.; Oks, E. Muonic-electronic negative hydrogen ion: Circular states. Can. J. Phys. 2013, 91, 715-721. [CrossRef]

5. Kryukov, N.; Oks, E. Super-generalized Runge-Lenz vector in the problem of two Coulomb or Newton centers. Phys. Rev. A 2012, 85, 054503. [CrossRef]

6. Fox, K. Classical motion of an electron in an electric-dipole field. II. Point dipole case. J. Phys. A General Phys. 1968, 1, $124-127$. [CrossRef]

7. Jones, R.S. Circular motion of a charged particle in an electric dipole field. Am. J. Phys. 1995, 63, 1042-1043. [CrossRef]

8. McDonald, K.T. Motion of a Point Charge near an Electric Dipole. 1996. Available online: http://www.hep.princeton.edu/ $\sim\{\}$ mcdonald/examples/dipole.pdf (accessed on 5 May 2020).

9. Oks, E. Oscillatory-precessional motion of a Rydberg electron around a polar molecule. Symmetry 2020, 12, 1275. [CrossRef]

10. Oks, E. Analytical results for the motion of a Rydberg electron around a polar molecule: Effects of a magnetic field of the arbitrary strength. Eur. Phys. J. D 2021, 75, 276. [CrossRef] 